\title{
Le territoire comme palimpseste lentique : une vision spatiale géohistorique des interactions hommes-milieux-plans d'eau
}

Territory as a lentic palimpsest : a geohistorical vision of Man-environmentwater bodies relations

Das Staatsgebiet als ein Wechselspiel von Entstehen und Vergehen stehender Gewässer: ein Blick auf die Wechselwirkungen zwischen Mensch, Umgebung und stehenden Gewässern aus räumlich-erdgeschichtlicher Perspektive

\section{Pascal Bartout}

\section{(2) OpenEdition}

\section{Electronic version}

URL: http://journals.openedition.org/rge/5723

DOI: $10.4000 /$ rge. 5723

ISSN: $2108-6478$

\section{Publisher}

Association des géographes de l'Est

\section{Printed version}

Date of publication: 22 April 2016

ISSN: 0035-3213

\section{Electronic reference}

Pascal Bartout, « Le territoire comme palimpseste lentique : une vision spatiale géohistorique des interactions hommes-milieux-plans d'eau », Revue Géographique de l'Est [Online], vol.56 / n¹-2 | 2016, Online since 25 August 2016, connection on 10 December 2020. URL : http://journals.openedition.org/ rge/5723; DOI : https://doi.org/10.4000/rge.5723

This text was automatically generated on 10 December 2020 .

Tous droits réservés 


\section{Le territoire comme palimpseste lentique : une vision spatiale géohistorique des interactions hommes-milieux-plans d'eau}

Territory as a lentic palimpsest : a geohistorical vision of Man-environmentwater bodies relations

Das Staatsgebiet als ein Wechselspiel von Entstehen und Vergehen stehender Gewässer: ein Blick auf die Wechselwirkungen zwischen Mensch, Umgebung und stehenden Gewässern aus räumlich-erdgeschichtlicher Perspektive

\section{Pascal Bartout}

\section{Introduction}

De multiples études scientifiques portant sur les politiques de gestion de l'eau tant à l'échelle française (LEMA de 2006) qu'européenne(DCE-2000) ont montré que l'état des connaissances limniques actuel était insuffisant pour l'efficience de celles-ci (McCormick, 2001, Moss, 2008, Dufour \& Piégay, 2009, Hatton-Ellis, 2010, Surridge \& Watson, 2012, Bartout \& Touchart, 2013, Finger-Stich, 2013, Bouleau \& Pont, 2014, Bartout, 2015a). Deux critères ressortent prioritairement pour caractériser ces biais des politiques de l'eau: le manque d'intégration de l'Homme et les connaissances très approximatives des localisations exactes des plans d'eau, notamment ceux d'origine anthropique. Or, ces deux critères constituent la variable discontinue du géosystème "plan d'eau ", complément spatial indispensable à la trame continue constituée du potentiel d'accueil lentique (Bartout, 2015b), afin de faire face aux enjeux environnementaux contemporains.

Pour intégrer les multiples caractéristiques sociétales des paysages lentiques français, l'entrée scalaire et fonctionnelle qui a été choisie correspond au concept de 
«territoire ». En effet, le terme de "territoire », en tant qu'espace de gestion, est « au cœur des préoccupations des scientifiques, des politiques mais également des acteurs économiques " (Moine, 2005), car il répond à un manque entre l'échelle de planification et le développement local. Traditionnellement, le territoire est enfermé dans des limites assez rigides de types administratifs dont la règlementation se fait l'écho. Pourtant, pour A. Moine (2005), "le territoire est une construction intellectuelle mouvante, évolutive, floue » reposant sur "l'appropriation par des groupes d'individus» etcomposé de sous-systèmes et de "relations multiples, notamment des boucles de rétroaction positives ou négatives ». Cette vision du territoire "recouvre une complexité qui demeure difficile à saisir, à cerner ", tout devenant territoire (Lévy \& Lussault, 2003). De fait, il est nécessaire de penser les territoires avec plusieurs niveaux d'agrégations d'acteurs (Vanier, 2008) et cette approche passe par l'espace géographique car il met en «relation les systèmes de représentation qui guident les sociétés dans l'appréhension qu'elles ont de leur environnement " (Moine, 2005).

3 Le concept de «territoire de l'eau » (Laganier \& Salvador, 2001) a semblé être un moyen de mettre en lumière l'aspect multiforme du territoire, comprenant une dimension naturelle mais aussi socialisée de l'espace par l'entremise de l'identité, du vécu, du paysage, des projets ou du risque (Bravard \& Pourtier, 2003). Sans cesse perfectionné dans son contenu et son contenant (Ghiotti, 2007, Bédoucha, 2011, Blouin-Gourbilière, 2013, Touchart, 2014, Bartout, 2015b), ce concept a permis d'assembler la dimension spatiale et l'anthroposystème au cœur des problématiques des bassin-versants, puis de milieux aux contours moins en lien avec les dynamiques hydrologiques, aboutissant au « territoire limnique ${ }^{1}$ » (Bartout, 2015b).

En s'intéressant au territoire et à ses acteurs, nous remettons sur le devant de la scène l'intérêt des sciences sociales en limnologie, point cher à son concepteur F.A. Forel (1892), sans qui la genèse de cet espace géographique, les processus l'affectant ou encore son caractère palimpseste, ne pourraient être perçus. Cet «œkoumène ${ }^{2}$ limnologique» (Bartout, 2015b) sera appréhendé grâce à la triple expertise géographique, à savoir temporelle, thématique et spatiale.

5 Cette compréhension de l'œkoumène limnologique présent et passé et de son impact sur les localisations des plans d'eau artificiels s'effectuera en deux temps. Tout d'abord, nous nous intéresserons aux groupes sociaux, aux choix politiques et aux raisons économiques ayant pu influer sur les variations temporelles et spatiales de plans d'eau. Ensuite, nous essaierons de matérialiser ces fluctuations historiques de plans d'eau à partir de l'analyse de supports cartographiques anciens, en adoptant une démarche multiscalaire aussi bien temporelle que spatiale, visant à faire émerger les lignes directrices de ces concentrations en allant du global vers le local.

\section{L'édification des plans d'eau artificiels : des lieux et des Hommes.}

6 Pour comprendre la genèse de ces territoires limniques, il faut se pencher sur les sociétés humaines qui les entourent. Celles-ci, en fonction de leurs besoins instantanés, ont fait naître, grandir, vieillir ou disparaître les plans d'eau en cherchant à tirer profits de la retenue d'eau ainsi constituée. Elles ont donc engendré des espaces d'influences et d'actions fluctuant dans le temps, déterminant des trajectoires paysagères (Clément, 1999) et des territoires limniques sans cesse réécrits. Comme le 
rappelle A. Corboz (2001), «le territoire n'est pas un emballage perdu ni un produit de consommation qui se remplace. Chacun est unique, d'où la nécessité de "recycler", de gratter une fois encore le vieux texte que les hommes ont inscrit sur l'irremplaçable matériau des sols, afin d'en déposer un nouveau, qui réponde aux nécessités d'aujourd'hui avant d'être abrogé à son tour ». Nous allons donc appréhender le territoire limnique comme une œuvre dont l'état présent peut laisser supposer faire apparaitre des traces des versions antérieures, c'est-à-dire comme un palimpseste lentique. Pour ce faire, nous allons adopter une démarche géohistorique.

7 Pour V. Capdepuy et G. Djament-Tran (2012), la géohistoire est un néologisme braudelien qui vise à "promouvoir une histoire intégrant le temps long du milieu sous l'influence de la géographie environnementaliste ". Ce terme connait un renouveau depuis les années 1990 grâce à des chercheurs comme C. Grataloup (1996), T. Kirat et A. Torre (2008) ou N. Jacob-Rousseau (2009) pour qui il est impossible de «penser la dimension spatiale des sociétés sans sa dimension temporelle et réciproquement » (Capdepuy \& DjamentTran, 2012) remettant en cause les logiques des triptyques histoire-passé-archives et géographie-présent-terrain (Wallerstein, 1991).

8 En hydrologie, certains scientifiques ont développé cette approche comme M. Franchomme (2008) dans le Nord de la France ou L. Lespez (2012) en Basse-Normandie, dans le but de comprendre les dynamiques propres des milieux humides non restituables par transfert de modèles. En limnologie, cette recherche à visée géohistorique est restée très cloisonnée entre champs disciplinaires historiques et géographiques et n'a pas produit d'informations de portée nationale. En effet, l'étude géohistorique des plans d'eau s'est avant tout focalisée sur l'étude des microsociétés (en histoire) dont l'étang est un élément économique et culturel marquant, ou sur les mutations paysagères (en géographie), paysage dont l'étang est une composante, mais, pour chacune, le territoire d'étude est toujours bien défini et délimité (par exemple, C. Benoît en 1992 pour les Dombes, B. Sajaloli en 1993 pour le Laonnois, C. Marache en 2007 pour la Double, R. Benarrous en 2003 et 2009 ou G. Bédoucha en 2011 pour la Brenne). Les leçons scientifiques tirées de ces études sont alors avant tout locales, mais le manque de travaux à plus petite échelle fait qu'on leur donne (trop ?) souvent un caractère national, aux conséquences parfois fâcheuses lorsqu'il s'agit de produire des modèles de gestion globalisés comme peuvent l'être lois et décrets.

9 Ainsi, peu d'études ${ }^{3}$ envisagent l'étang comme un marqueur géographique et historique à l'échelle de plusieurs territoires limitrophes ou pas. Or, une compréhension plus globale de ce qui fait aujourd'hui l'étang passe par la compréhension de ce qui le faisait avant et par la localisation des milieux dans lesquels les Hommes avaient choisi de l'accueillir.

10 Historiquement, si une multitude d'écrits locaux (Kolar, 1991, Durand, 1993, Dérex, 2001, Benarrous \& Marinval, 2003, Miras, 2004, Germain, 2005, Cassan, 2006, Landou, 2006) font état de la présence d'étangs en France dès l'époque gallo-romaine, la véritable structuration stagnustre fruit d'une réflexion collective visant à l'aménagement d'un territoire est le fait des moines et des seigneurs laïcs. La prégnance progressive de la religion catholique dans la vie et les mœurs de l'époque ainsi que le pouvoir économique conféré par l'octroi de privilèges quant à l'affectation des sols vont permettre à ces catégories sociales d'exercer une mainmise lentique qui va durer du Moyen-âge à la Renaissance, permettant une continuité fonctionnelle et spatiale des étangs. La multiplication des acteurs imbriqués dans le processus 
stagnustre à partir du tournant de l'absolutisme (début $17^{\text {ème }}$ siècle) et les multiples soubresauts techniques, économiques et sociologiques occasionnés par les mutations sociétales au cours des deux derniers siècles vont avoir raison de ce modèle ancestral et multiplier les réponses stagnustres locales, moins organisées.

L'étang (et le territoire d'étangs) reflète un choix ponctuel de la société pour ce type d'aménagement car il répond au mieux aux exigences du temps. La création d'étangs répond donc à l'un des deux impératifs suivants : un choix de structuration durable ou une étape vers la bonification des terres. Ces va-et-vient temporels sont avant tout liés à des choix ponctuels de la société pour ce type d'aménagement car il répond au mieux aux exigences du temps, des territoires et des groupes sociaux qui les possèdent.

Extrêmement nombreux, les étangs anciens comportaient un certain nombre de caractères. Ils étaient pour la plupart polyfonctionnels. Contribuant de façon décisive à l'alimentation humaine aussi bien par la production de protéines que par la production d'énergie si nécessaire pour transformer les produits agricoles, ils mettaient en valeur les terres ingrates. Aussi ne fut-il jamais question pour les sociétés paysannes de se priver des potentialités de richesse des étangs. Seules les élites les ont sacrifiés au profit de cultures plus rentables comme la céréaliculture en Brie, puis les mutations économiques qui dépeuplèrent les régions peu adaptées aux changements opérés vont rayer les étangs de la carte de certains territoires. Comme le rappelle J-M. Dérex (2001), au plan national, « la révolution donna le premier coup de semonce, ... mais sur le long terme, les causes étaient ailleurs ».

Pour autant tout n'est pas si négatif car certains étangs et régions d'étangs ont subsisté en s'adaptant aux contextes et modes nouveaux, telle la chasse si prisée par la bourgeoisie et l'aristocratie qui a donné une nouvelle dimension lentique à des territoires comme la Sologne (pour Paris) et les Dombes (pour Lyon).

Si l'on dresse une synthèse physique et sociale des régions d'étangs au cours des 800 dernières années en fonction des paramètres développés jusqu'à présent, nous pouvons proposer le tableau 1 suivant où chaque groupe social va privilégier le milieu géographique répondant le plus à ses attentes du moment. La continuité stagnustre, si elle existe, est donc le fait de la perpétuation d'une rentabilité de l'étang, que ce soit par une classe ou la succession de classes sur un même lieu.

\begin{tabular}{|c|c|c|c|c|c|c|c|c|c|}
\hline \multirow{2}{*}{$\begin{array}{c}\text { Classe } \\
\text { sociétale }\end{array}$} & \multirow{2}{*}{$\begin{array}{c}\text { Période } \\
\text { préférentielle }\end{array}$} & \multicolumn{6}{|c|}{ Fonction recherchée } & \multicolumn{2}{|c|}{ Lieux préférentiels d'édification } \\
\hline & & Pêche & Chasse & Culture & $\begin{array}{l}\text { Irrigation } \\
\text { Elevage }\end{array}$ & $\begin{array}{l}\text { Force } \\
\text { motrice }\end{array}$ & Détente & $\begin{array}{c}\text { Plaines et } \\
\text { dépressions } \\
\text { marécageuses }\end{array}$ & $\begin{array}{c}\text { Fonds de vallée } \\
\text { Têtes de } \\
\text { bassins }\end{array}$ \\
\hline Moines & $13^{\mathrm{eme}}-18^{\mathrm{e} m e}$ & * & & $*$ & & & & * & \\
\hline Seigneurs & $14^{\mathrm{e} m e}-19^{\mathrm{e} m e}$ & * & * & * & & * & & * & $*$ \\
\hline Bourgeois & Depuis 17ème & * & $*$ & * & & & & * & \\
\hline Paysans & $19^{\dot{m} m}-20^{\dot{m} m e}$ & * & & * & * & & & & $*$ \\
\hline $\begin{array}{l}\text { Néo- } \\
\text { ruraux }\end{array}$ & Depuis 1960 & * & & & & & * & & ${ }^{*}$ \\
\hline
\end{tabular}

Tableau 1 : Sites et fonctions préférentiels des « faiseurs " d'étangs au cours des âges.

D'après ce tableau, il existe une relative continuité historique entre les moines (ainsi que les seigneurs) et les bourgeois qui les supplantent dans les mêmes lieux. Pour autant, les logiques animant ces populations sont contraires, expliquant en partie la rupture brutale observée lors du passage de témoin. En effet, comme le rappelle J.-R. Pitte (1989), si les moines se sont installés au cœur de marais, c'est en premier lieu pour être éloignés de la civilisation et de ses tentations matérielles. Grâce à sa mainmise sur la société, le clergé va pouvoir rentabiliser les importants travaux d'assainissement 
opérés, ayant conduit entre autres à la création de retenues d'eau pour l'empoissonnement de l'étang afin de respecter les périodes de jeûne très nombreuses (par exemple 206 jours au $16^{\text {ème }}$ siècle pour J.B. Rougier de la Bergerie, 1819). La pratique complémentaire de l'agriculture sur ces terres peu fertiles a conduit les moines à valoriser au maximum l'étang grâce à la rotation des mises en valeur connue sous le nom d'assec et d'évolage. Le fort potentiel financier tiré de la pisciculture a conduit certains seigneurs à investir les marais pour en tirer des revenus substantiels, en plus de la protection que pouvait constituer la retenue. C'est cet aspect financier qui va conduire la bourgeoisie à investir les lieux d'étangs, tout d'abord en complément des deux classes privilégiées, puis en remplacement de l'un d'eux suite aux déboires financiers de certains seigneurs laïcs, enfin en achetant les terres lors de la vente des Biens Nationaux. Ce changement majeur ne prendra guère plus d'un siècle (le $\left.18^{\text {ème}}\right)$ tout comme il touchera les fonds de vallée et les têtes de bassin colonisés de manière moins dense que les marais par les étangs, et ce même si l'antériorité de la présence est avérée.

16 La mainmise de la bourgeoisie et de la paysannerie aisée sur les terres au tournant $18^{\text {ème }}$ $-19^{\text {ème }}$ a un impact direct sur la santé des étangs : seule la rentabilité est recherchée et si l'étang apparaît comme un piètre rendement financier, il est détruit et transformé. C'est ce qui arrive justement en 1793 avec le décret du 14 Frimaire An II, dit « décret sur les assèchements d'étangs ", influencé par les théories agraires de l'Abbé Rozier, moment du premier inventaire national des étangs. Nombre d'étangs sont ainsi détruits (J.B. Rougier de la Bergerie parle «des dix-huit vingtièmes » à l'échelle nationale à la page 194 de son Manuel des étangs, mais la remarque vaut "pour un siècle», sans que nous puissions infirmer ou confirmer ses dires) ou réduits surfaciquement pour laisser place aux cultures. Cependant, la plupart des terres dévolues jusque là aux étangs sont agronomiquement pauvres et ne peuvent supporter plusieurs récoltes successives sans fertilisants. Ce constat est vite dressé par les nouveaux propriétaires des terres et deux logiques vont s'affronter :

17 - la logique bourgeoise : ayant des capitaux et acquis des terres proches des villes, les bourgeois vont massivement investir dans les engrais pérennisant la destruction des étangs (comme en Brie d'après J.-M. Dérex, 2001) ou, là où l'hydromorphie était trop importante, ils vont réaménager progressivement l'étang en un lieu de chasse prisé par les grands de ce monde (comme en Sologne ou dans les Dombes);

18 - la logique paysanne : ayant des capitaux moindres, ils vont souvent être à l'origine du maintien ou de la reconstruction des étangs qui constituaient un élément essentiel de l'aménagement agraire des campagnes, notamment par la présence de moulins et de systèmes perfectionnés d'irrigation comme les levades limousines.

19 Malgré les crises hygiénistes qui vont faire fondre les superficies en eau jusqu'au début $\mathrm{du} 20^{\mathrm{ème}}$ siècle, la mise en valeur bourgeoise des régions d'étangs va résister aux mutations socio-économiques des $19^{\text {ème }}$ et $20^{\text {ème }}$ siècles, perpétuant les localisations d'étangs au sein des espaces marécageux qui vont être hérissés de voies routières (de 1849 à 1877 en Sologne et de 1860 à 1872 en Brenne pour A. Vacher, 1908). Les sites de fond de vallée et les têtes de bassin vont eux souffrir des révolutions agricoles et surtout de l'exode rural, au point même de voir pratiquement disparaître l'étang de secteurs géographiques jusque là très prisés (Nord-Ouest du Massif Central, Est du Massif Armoricain) (Balabanian \& Bouet, 1989, Bartout, 2012). Néanmoins, cet abandon des campagnes va paradoxalement constituer un vivier pour le renouveau des étangs 
observé depuis les années 1960. En effet, les néo-ruraux investissent les campagnes où les prix du foncier sont peu élevés et cherchent à aménager un lieu convivial pour eux et leur famille. Cette société de loisirs débarquant dans les campagnes va être à l'origine de la colonisation de tous les espaces propices à l'édification d'une digue sur un petit territoire (principalement les pentes) souvent même avant l'écoulement permanent du cours d'eau ( $80 \%$ des étangs actuels en Limousin d'après P. Bartout, 2010). Ce phénomène va toucher également les deux grands sites d'édification d'étangs depuis les origines que sont les plaines et dépressions marécageuses, mais la nature de la propriété foncière va faire que ce sont avant tout les têtes de bassin qui vont " profiter » de ce renouveau (les fonds de vallée encaissés vont quant à eux être très souvent délaissés) comme le montre la figure 1.

Figure 1 : Schémas théoriques de la spatialisation des plans d'eau à l'intérieur d'un bassin-versant de taille régionale, du Moyen-âge aux années $1950(\mathrm{~A})$ et depuis la deuxième moitié du XXème siècle (B) (d'après Bartout P., 2012)

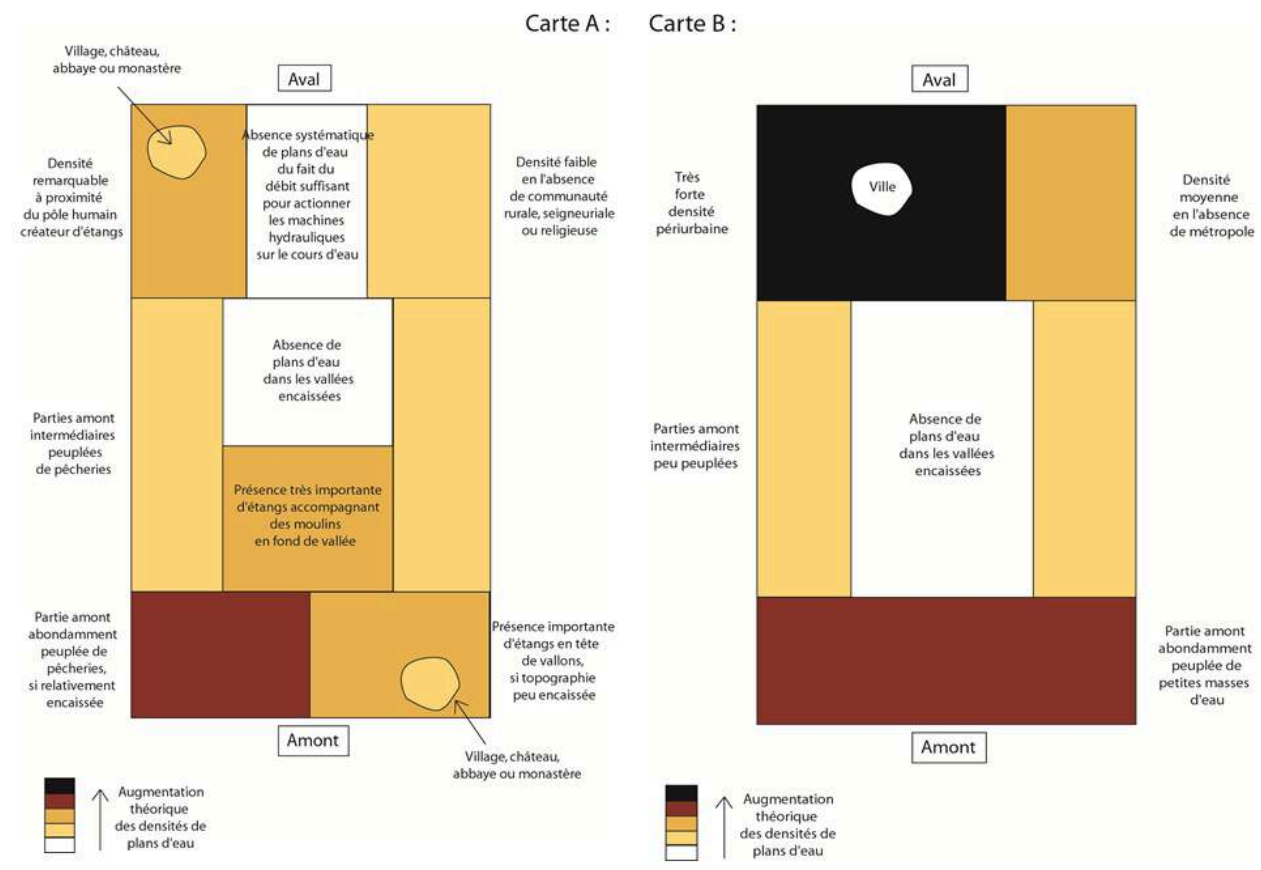

Toutes ces phases d'aménagements et de destructions de plans d'eau ont laissé des traces dans les paysages et se doivent d'être quantifiées au plus près. Grâce aux analyses de cartes, de textes et de plans anciens, nous allons mener une forme d'archéologie du paysage avec des outils géographiques ceci afin de mieux comprendre les situations lentiques actuelles et envisager les territoires limniques comme palimpsestes.

21 Les variations de valorisations de l'étang dans les sociétés laissent entendre des fluctuations de leurs nombres et de leurs lieux d'implantation au cours de l'histoire plus ou moins récente. Comme le rappelle M. Lutz (2001), le «paysage produit par les étangs, s'il peut paraître figé en comparaison à d'autres paysages agraires, peut donc évoluer, jusqu'à la complète disparition des étangs ".

22 Grâce aux SIG et à la possibilité offerte de localiser parfaitement chaque étang et ses contours $^{4}$, la science stagnustre peut profiter aujourd'hui d'analyses statistiques à différentes échelles géographiques, dont celles administratives. Ainsi, il est possible de 
dresser des cartes de stagnucité ${ }^{5}$ ou de densité d'étangs, quelle que soit l'échelle retenue, comme le montrent les figures 2 et 3 ci-après à l'échelle communale.

Figure 2 : La stagnucité par commune en France en 2005.

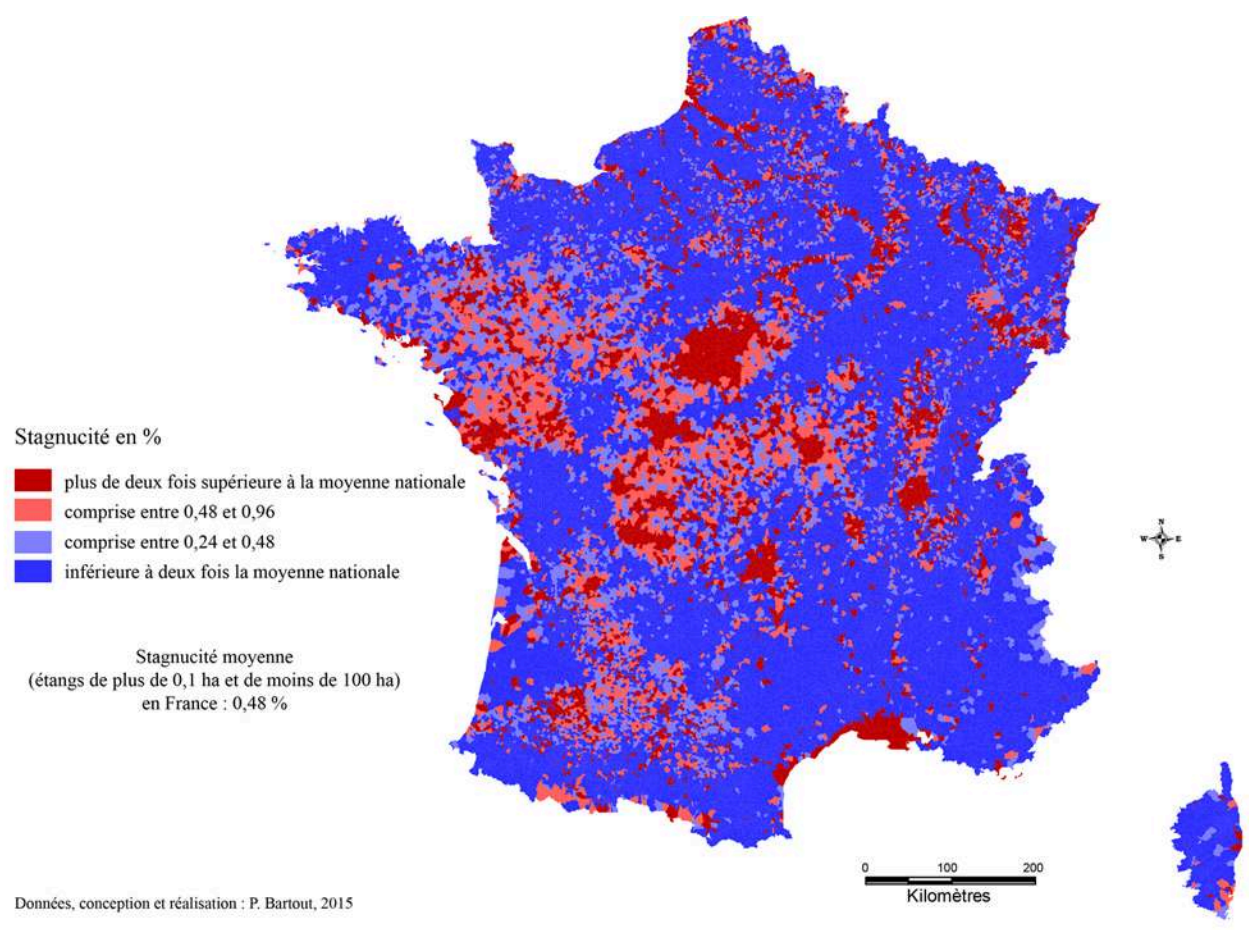

Figure 3 : La densité d'étangs par commune en France en 2005.

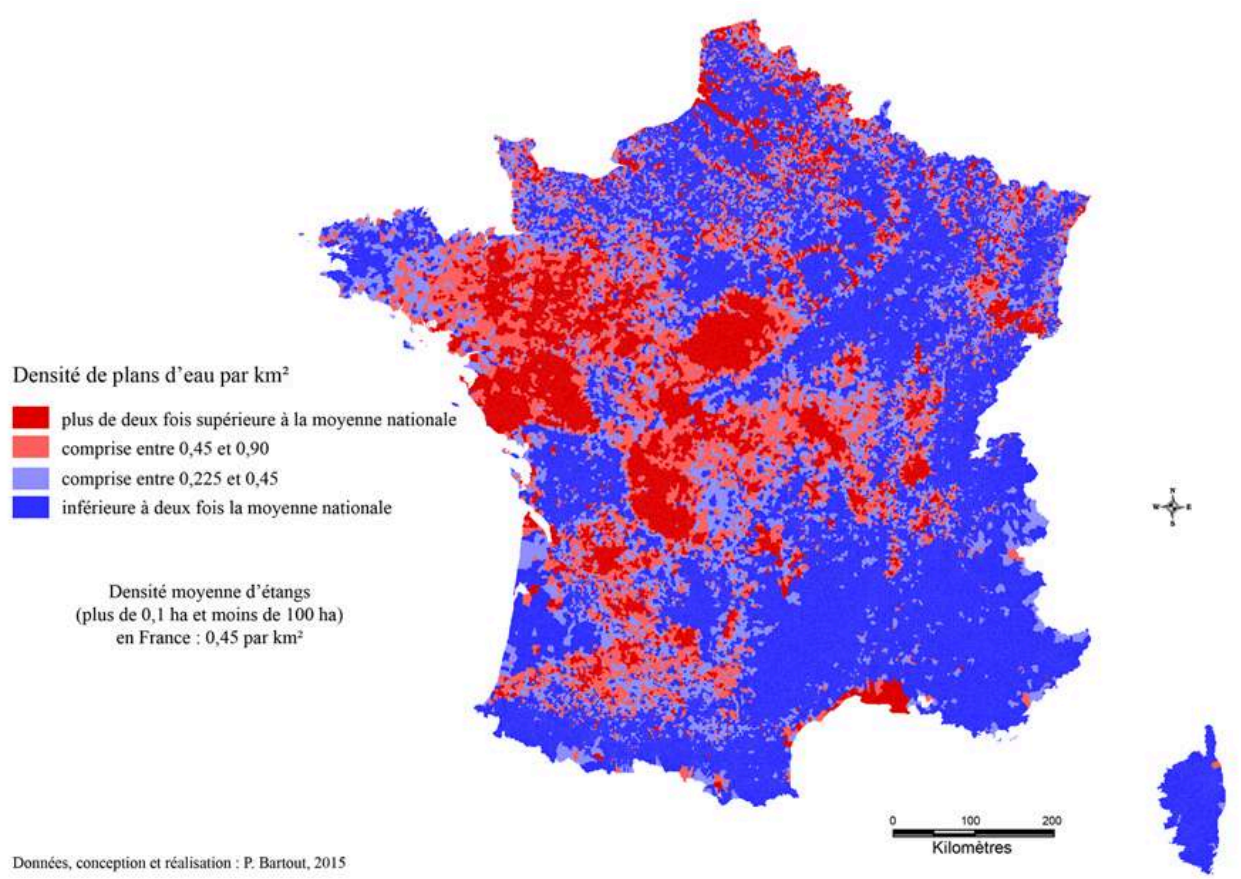

23 Cependant, si ces cartes permettent d'identifier actuellement des lieux préférentiels d'implantation comme la Sologne, les marais et bocages vendéens, la Camargue, les Dombes, le Forez, le rebord nord-ouest du Massif Central, la Champagne-Humide, le 
Sundgau ou encore la vallée de la Somme, elles ne reflètent pas toute la diversité limnique artificielle, et le manque de comparaison temporelle par absence de données historiques équivalentes ne permet pas de comprendre tous les leviers de la résilience lentique. Pour cela, il est nécessaire d'entreprendre des travaux comparatifs dont l'échelle d'étude correspond aux échelles précédemment étudiées, même si elles n'offrent pas la finesse d'analyse potentielle actuelle (Bartout, 2015c).

Grâce à la mise à jour de travaux statistiques anciens dont les limites méthodologiques sont connues (Bartout, 2015c), il est désormais possible de dresser les grandes lignes de l'évolution lentique (essentiellement stagnustre) à l'échelle du territoire français depuis la fin du $18^{\text {ème }}$ siècle et ainsi de mieux comprendre les dynamiques temporelles au sein d'espaces géographiques identifiés, qu'ils soient locaux, régionaux ou nationaux. Afin de faire apparaitre ces fluctuations historiques sur un plan spatial, les concentrations lentiques seront appréhendées de deux manières (surfaciques et numériques) à trois moments de l'histoire : 1793, 1834 et 2005. Ces dates correspondent aux trois recensements nationaux jugés fiables méthodologiquement (Bartout, 2015c), à savoir ceux réalisés sous la direction du baron J.B. Rougier de la Bergerie en 1819 (date effective de l'inventaire: 1793) et en 1834 concernant les seuls étangs et lagunes littorales, et celui de P. Bartout et L. Touchart en 2013 (date effective de l'inventaire : 2005) portant sur l'ensemble du corpus lentique.

Ces données statistiques vont permettre ainsi de dresser la carte d'identité de la France lentique à trois moments de l'histoire au cours des deux derniers siècles et de tenter d'en comprendre les mutations spatiales. En dressant un parallèle avec les mutations sociétales qui ont parcourues les $19^{\text {ème }}$ et $20^{\text {ème }}$ siècles, nous lierons spatialité et temporalité en mettant à jour les lieux d'implantation d'étangs les plus réactifs aux évolutions de la société et ceux qui se perpétuent quelles que soient les vicissitudes économiques, politiques et culturelles.

\section{A. Quelle France lentique en 1793, 1834 et 2005 ?}

26 Construit en France le plus souvent de la main de l'Homme pour répondre à des besoins anthropiques, le plan d'eau est appréhendé différemment selon les époques et les lieux dans lesquels il s'insère. En cela, il est intimement lié à l'économie locale et aux fluctuations historiques de celle-ci (Bartout \& Bernard, 2007, Bernard, 2008, Bartout, 2010). L'argument premier de sa définition oscille donc tour à tour entre sa fonction économique (pêche, chasse, agriculture, force motrice, loisirs ...) et son fonctionnement physico-chimique (faune et flore, zonations horizontales et verticales, insertion dans le continuum fluvial ...) (Genest, 2007).

Nous avons donc tenu compte de ces nuances sémantiques pour permettre d'élaborer des cartographies matérialisant les fluctuations quantitatives dudit objet " plan d'eau ». Or, l'intérêt du support cartographique est qu'il peut transgresser sans problème les barrières du langage, c'est-à-dire faire fi de savoir ce qui est, par exemple, «étang » ou ne l'est pas. Pour cela, une représentation à l'échelle cartographique la plus fine possible permet de recenser, localiser et renseigner tous types de retenues d'eau, sans distinction linguistique préconçue, et offre à celui ou celle qui l'étudie des années plus tard la possibilité de scinder les résultats issus de ces travaux en fonction de l'acception du jour (Bartout, 2011). 


\section{Qu'entend-on par « plan d'eau » à chaque date sélectionnée?} comprendre ce que le ou les auteurs entendaient par "plan d'eau ». Dans le cas des travaux de J.B. Rougier de la Bergerie, il s'agit de la présence de la représentation d'un plan d'eau sur la carte de Cassini et du décompte personnel sur quelques départements (Cassan, 2006, Bartout, 2011) puis de sa mise à jour grâce au cadastre napoléonien (pour l'inventaire de 1834). Compte tenu de l'intérêt foncier porté aux étangs au $19^{\text {ème }}$ siècle, seuls ces types de plans d'eau ont été recensés en 1793 puis en 1834. Pour autant, tous ne sont pas comptabilisés en raison notamment du faible degré de fiabilité du support d'inventaire (Pelletier, 2002) et cette absence de repère strict ne permet pas de sélectionner les plans d'eau correspondant dans le référentiel lentique de tous les plans d'eau de plus de 0,01 ha en 2013.

En conséquence, cette étude a porté sur l'ensemble des plans d'eau recensés quelle que soit l'époque, et les trois inventaires ont été comparés en fonction du plus petit dénominateur spatial commun, à savoir l'échelle régionale, afin de tenir compte de ce biais méthodologique.

\section{Les données surfaciques des inventaires}

Si dans une logique de continuités hydrologiques et sédimentaires, il est appréciable de produire une comparaison historique à l'échelle de bassin-versants, la constitution des deux bases de données de 1793 et 1834 ne nous le permet pas. De fait, celles-ci étant construites à partir de l'échelon administratif régalien qui a perduré aux vicissitudes historiques des deux derniers siècles, à savoir le département (à quelques exceptions près comme la Sologne), c'est à cette échelle et à celle de la région administrative (où les données départementales peuvent être compilées) que l'approche a été effectuée pour visualiser des comportements lentiques sur une échelle temporelle relativement longue.

Notre examen de la question quantitative des plans d'eau débute en 1793 au moment où l'auteur de cet inventaire, J.B. Rougier de la Bergerie (1819), note que «les 2/3 au moins des étangs [antérieurs à 1793] n'existent plus». Cela signifie pour lui qu'il y avait "plus d'un million d'arpens en étangs, pendant le règne le plus absolu des moines et des prêtres", alors que son tableau paru en 1819 dans son «Manuel des Etangs» ne fait état que de 308000 arpents.

Le tableau 2 ci-après compile les informations recueillies lors des trois recensements de plans d'eau en ayant pris soin de modifier les arpents en ha. Nous allons décortiquer ces données en séparant l'analyse dynamique temporelle de celle statique de chaque recensement. 
Tableau 2 : Comparaison des superficies départementales d'étangs entre les trois inventaires

\begin{tabular}{|c|c|c|c|c|c|c|c|}
\hline Département & 1793 & 1834 & $2005^{1}$ & Département & 1793 & 1834 & 2005 \\
\hline Ain & 13537 & 18858 & 11270 & $\begin{array}{c}\text { Lozère } \\
\text { Minne-et. }\end{array}$ & $\begin{array}{r}31 \\
4182\end{array}$ & 2 & $\begin{array}{l}4433 \\
4577\end{array}$ \\
\hline $\begin{array}{l}\text { AAsine } \\
\text { Allier }\end{array}$ & $\begin{array}{l}\begin{array}{l}2958 \\
1734\end{array}\end{array}$ & $\begin{array}{l}1126 \\
4759\end{array}$ & 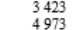 & $\begin{array}{l}\text { Maine-ett-Lit } \\
\text { Manche }\end{array}$ & $\begin{array}{l}\begin{array}{l}4182 \\
1683\end{array}\end{array}$ & $\begin{array}{r}1327 \\
359 \\
\end{array}$ & $\begin{array}{l}\begin{array}{l}5777 \\
1628\end{array} \\
-\end{array}$ \\
\hline Alpes (Basses) (de & & 2 & 754 & Marne & 4845 & 4001 & 4131 \\
\hline $\begin{array}{l}\text { Haute-Provence) } \\
\text { Alpes (thutes }\end{array}$ & & 2 & & Mame (Haute) & & 702 & \\
\hline Alpes Maritimes & & & 394 & $\begin{array}{l}\text { Manne (rature) } \\
\text { Mayenne }\end{array}$ & $\begin{array}{l}2550 \\
2550\end{array}$ & 1264 & $\begin{array}{l}1174 \\
3051\end{array}$ \\
\hline Ardeche & & 19 & 586 & Meurthe (et & 2252 & 3590 & \\
\hline Ardennes & 979 & 325 & 1229 & $\begin{array}{l}\text { Moselle) } \\
\text { Meuse }\end{array}$ & 3315 & 2272 & \\
\hline Ariège & 1530 & 881 & 1487 & Morbihan & 1224 & 2059 & 2995 \\
\hline Aube & 2950 & 1429 & 2279 & Moselle & 1712 & 772 & 4953 \\
\hline Aude & 3570 & 5152 & 2605 & Nièvre & 3570 & 3023 & 2636 \\
\hline Aveyron & 81 & 58 & 1139 & Nord & 918 & 475 & 2680 \\
\hline $\begin{array}{l}\text { Bouches-du-Rhône } \\
\text { Calvados }\end{array}$ & $\begin{array}{l}408 \\
765\end{array}$ & $\begin{array}{r}11565 \\
101\end{array}$ & $\begin{array}{l}9074 \\
1216\end{array}$ & $\begin{array}{l}\text { Oise } \\
\text { Ome }\end{array}$ & 1275 & 386 & $\begin{array}{r}2513 \\
1655\end{array}$ \\
\hline $\begin{array}{l}\text { Calvados } \\
\text { Cantal }\end{array}$ & $\begin{array}{l}763 \\
561\end{array}$ & $\begin{array}{l}154 \\
154\end{array}$ & $\begin{array}{l}1216 \\
5722\end{array}$ & $\begin{array}{l}\text { Ome } \\
\text { Pass-de-Calais }\end{array}$ & $\begin{array}{r}3823 \\
51\end{array}$ & $\begin{array}{r}1160 \\
681\end{array}$ & $\begin{array}{l}1623 \\
2466\end{array}$ \\
\hline Charente & 1785 & 410 & 1910 & Puy-de-Dôme & 2295 & 1133 & 3405 \\
\hline Charente-Inférieure & 867 & 73 & 1395 & Pyránées (Basses) & & 186 & 1492 \\
\hline $\begin{array}{l}\text { (Manitime) } \\
\text { Cher }\end{array}$ & 2397 & 3554 & 5827 & $\begin{array}{l}\text { (Atlantiques) } \\
\text { Pyrénes (Hautes) }\end{array}$ & & 57 & \\
\hline Corrèza & 2448 & 1077 & 2790 & Pyrénées-Orientales & 765 & 4749 & 1603 \\
\hline Corse-du-Sud & & & 507 & Rhin (Bas) & 1173 & 78 & 2183 \\
\hline Haute-Corse & & & 609 & Rhin (Haut) & 1071 & 1495 & 1692 \\
\hline & 2075 & 2577 & 3070 & Rhốne & $4845(E)$ & 40 & 722 \\
\hline $\begin{array}{l}\text { Côtes du Nord } \\
\text { (d'Armor) }\end{array}$ & 1326 & 1152 & 1888 & Saône (Haute) & & 1687 & 3035 \\
\hline Creuse & 3723 & 2747 & 3662 & Saône-et-Loire & 4952 & 4995 & 5250 \\
\hline Dordogne & 2303 & 706 & 4017 & Sarthe & 2703 & 819 & 3026 \\
\hline Doubs & 612 & 272 & 959 & $\begin{array}{l}\text { Savoie } \\
\text { Sald }\end{array}$ & & & $\begin{array}{c}1333 \\
589\end{array}$ \\
\hline Eure & 137 & $\begin{array}{r}33 \\
205\end{array}$ & $\begin{array}{r}444 \\
1747\end{array}$ & $\begin{array}{l}\text { Savoie (Haute) } \\
\text { Seine }\end{array}$ & & $16(\mathrm{C})$ & 589 \\
\hline Eure-et-Loir & 1646 & 399 & 1841 & Seine-Infférieure & 10 & 16 & 1810 \\
\hline Finistere & 255 & 1041 & 1023 & $\begin{array}{l}\text { (Maritime) } \\
\text { Seine-et-Marme }\end{array}$ & 911 & 646 & 3991 \\
\hline Gard & & 2954 & 5331 & Yvelines & 193 (B) & 287 (B) & 1130 \\
\hline Garonne (Haute) & & & 3177 & Sèvres (Deux) & $\begin{array}{c}1938 \\
561\end{array}$ & 1015 & 2979 \\
\hline $\begin{array}{l}\text { Gers } \\
\text { Givon }\end{array}$ & 918 & $\begin{array}{r}289 \\
3699 \\
-69\end{array}$ & $\begin{array}{l}3830 \\
4583 \\
4583\end{array}$ & $\begin{array}{c}\text { Somme } \\
\text { Tam }\end{array}$ & ${ }_{20}^{561}$ & 1018 & $\begin{array}{r}4546 \\
1936\end{array}$ \\
\hline $\begin{array}{l}\text { Gronch } \\
\text { Herau }\end{array}$ & 561 & $\begin{array}{r}3609 \\
11713\end{array}$ & $\begin{array}{l}4885 \\
2847\end{array}$ & Tam-et-Garomne & & & $\begin{array}{l}1936 \\
1934\end{array}$ \\
\hline IIle-et-Vilaine & 2295 & 2905 & 4907 & Var & & & 856 \\
\hline Ind & 1780 & 10580 & 12194 & Vau & & & 522 \\
\hline Indre-et-Loire & 2295 & 97 & & & 3060 & 566 & 6776 \\
\hline 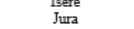 & $\begin{array}{l}918 \\
935\end{array}$ & $\begin{array}{l}1037 \\
1505\end{array}$ & $\begin{array}{l}2764 \\
1949\end{array}$ & $\begin{array}{l}\text { Vienne } \\
\text { Viemne (Haute) }\end{array}$ & $\begin{array}{l}2754 \\
4503\end{array}$ & $\begin{array}{r}913 \\
2328\end{array}$ & $\begin{array}{l}3136 \\
5142\end{array}$ \\
\hline Lande: & & 11034 & 3322 & Vo & 812 & 1042 & 1595 \\
\hline Loir-et-C & 9149 (A) & 9812 & 10205 & Youne & 6960 & 1336 & 2827 \\
\hline Loi & 4845 (E) & 3768 & 2856 & Territoire-de-Belfort & (D) & (D) & 1199 \\
\hline $\begin{array}{l}\text { Loire (Haute) } \\
\text { Loir-Inficieure }\end{array}$ & $\begin{array}{l}1173 \\
6868\end{array}$ & $\begin{array}{r}181 \\
5515\end{array}$ & $\begin{array}{r}524 \\
4184\end{array}$ & $\begin{array}{l}\text { Essonne } \\
\text { Hante }\end{array}$ & 193 (B) & 287 (B) & 823 \\
\hline & & & & Hauts-de-Seine & & & \\
\hline Loiret & 9149 (A) & 4514 & 7299 & Seine-Saint-Denis & & 16 (C) & 39 \\
\hline $\begin{array}{l}\text { Lot } \\
\text { Lot-et Garome }\end{array}$ & & & $\begin{array}{r}441 \\
2959\end{array}$ & $\begin{array}{l}\text { Val-d } \\
\text { Val }\end{array}$ & 193 (B) & $\begin{aligned} 16 \text { (C) } \\
287(\mathrm{~B})\end{aligned}$ & $\begin{array}{l}115 \\
377\end{array}$ \\
\hline & & & & $\frac{\mathrm{Tall}}{\mathrm{TO}}$ & 157192 & 177168 & 50694 \\
\hline
\end{tabular}

(A) : Loir-et-Cher + Loiret. (B) : Essonne, Val-d'Oise et Yvelines (plus quelques communes des Hautsde-Seine, de Seine-Saint-Denis et du Val-de-Marne) sous le nom de Seine-et-Oise. (C) : Seine, Hauts-deSeine, Seine-Saint-Denis et Val-de-Marne sous le nom de Seine. (D) : Le Territoire-de-Belfort faisait partie du Haut-Rhin. (E) : Rhône et Loire sous le nom de Rhône-et-Loire

(D’après Rapport général sur les étangs, J.B. Rougier de la Bergerie et P. Bartout, inédit).

33 A l'échelle française, plusieurs informations principales ressortent de ces données. Tout d'abord et contrairement à ce que certaines références bibliographiques laisseraient penser (par exemple, "l'assèchement des 6/11 ${ }^{\text {ème }}$ de la surface des étangs entre 1792 et 1878 " en Dombes pour C. Benoît, 1992, ou encore le passage d'une superficie des étangs français de 210000 ha en 1792 à 100000 ha au début du $19^{\text {ème }}$ siècle pour P. Reyt, 1998), la situation des étangs est très complexe dans la première partie du $19^{\text {ème }}$ siècle, faite de va-et-vient.

Malgré les assèchements effectués, certaines régions gardent une forte prégnance des étangs avec environ $20 \%$ du sol inondé en Brenne ou en Sologne et nous assistons à une très légère hausse des superficies en eau entre 1793 et 1834 (plus 20000 ha). La stagnucité moyenne passe ainsi de $0,291 \%$ à $0,322 \%$, même si cette augmentation est avant tout liée à la prise en compte des « étangs » littoraux (Aude, Bouches-du-Rhône, Gard, Gironde, Hérault, Landes, Pyrénées-Orientales). Si on élimine de l'inventaire ces sept départements (soit 44716 ha en plus), on constate en fait une légère baisse des superficies en eau (environ $25000 \mathrm{ha}$ ), en lien avec l'application du décret du 14 Frimaire an II qui visait à détruire les étangs ou les réduire à la taille d'un « arpens ». Cependant, la réponse départementale est variée puisque vingt départements (autres que ceux déjà énoncés) augmentent leurs superficies en eau, parfois notablement comme l'Allier, alors que quarante-six voient leurs superficies baisser (parfois de manière impressionnante comme dans l'Yonne ou le Bas-Rhin avec $95 \%$ de réduction). 
Entre 1793 et 2005 ou entre 1834 et 2005, les informations retirées sont statistiquement plus complexes mais pour autant plus tranchées: il s'agit d'une très forte hausse des superficies en eau. Celles-ci sont multipliées par 3 entre 1793 et 2005, la limnicité étendue ${ }^{6}$ moyenne atteignant alors la valeur de $0,823 \%$, et, si l'on applique cette méthodologie aux seuls « étangs » de 2005, à savoir tous les plans d'eau de superficie comprise entre 0,1 ha et 100 ha, la stagnucité augmente de $50 \%$ pour atteindre $0,45 \%$.

Cette hausse observée tient avant tout à l'effet de mode que sont les loisirs, même si 22 départements sur 96 ne réagissent pas de cette manière : pour quatorze d'entre eux, le maximum surfacique intervient en 1793 et pour les huit autres en 1834. Il est intéressant d'observer que ceux concernés par l'année 1834 sont à deux exceptions près des départements littoraux : la chute de la limnicité étendue peut alors être le fait de l'assainissement des marais littoraux comme J.L. Abbé (2007) le présente en Languedoc.

37 Les départements affichant des valeurs maximales en 1793 sont moins sujets à ces biais statistiques à l'exception peut-être de la Loire-Atlantique. Ils se situent majoritairement dans le nord-est de la France (Meuse, Haute-Marne, Haute-Saône) mais surtout à proximité d'agglomérations importantes (Marne, Aube et Yonne pour Paris, Loire pour Lyon) ou sont restés très profondément ruraux (Orne, Manche, Creuse, Haute-Loire), avec parfois des vicissitudes historiques funestes pour l'Eglise (Creuse). Ces trois derniers points sont défavorables foncièrement quant à la pérennisation des étangs par le détournement des capitaux locaux ou la faiblesse de ceux-ci ne permettant pas d'assumer les coûts prohibitifs des étangs.

Appliqués à l'échelle régionale (tableau 3), ces chiffres amplifient l'analyse géographique puisque trois régions ont connu leur maximum de limnicité étendue en 1793, toutes situées à proximité de la région parisienne (Basse-Normandie, Bourgogne, Champagne-Ardenne), et deux en 1834, correspondant au développement de la métropole lyonnaise (Rhône-Alpes) et au drainage d'un certain nombre de marais maritimes (Languedoc-Roussillon).

Tableau 3 : La limnicité étendue régionale au moment des trois inventaires.

\begin{tabular}{|c|c|c|c|c|c|c|c|c|c|}
\hline & \multicolumn{3}{|c|}{$\mathbf{1 7 9 3}$} & \multicolumn{3}{|c|}{$\mathbf{1 8 3 4}$} & \multicolumn{3}{|c|}{$\mathbf{2 0 0 5}$} \\
\hline Région & Rang & $\begin{array}{c}\text { Limnicité } \\
\text { étendue }\end{array}$ & Ha & Rang & $\begin{array}{c}\text { Limnicité } \\
\text { étendue }\end{array}$ & Ha & Rang & $\begin{array}{c}\text { Limnicité } \\
\text { étendue }\end{array}$ & Ha \\
\hline Limousin & 1 & 0,63 & 10674 & 7 & 0,36 & 6152 & 2 & 0,68 & 11595 \\
Pays-de-la-Loire & 2 & 0,60 & 19363 & 9 & 0,29 & 9491 & 3 & 0,67 & 21616 \\
Centre-Val-de-Loire & 3 & 0,59 & 23267 & 2 & 0,78 & 30556 & 1 & 1,04 & 40977 \\
Bourgogne & 4 & 0,56 & 17757 & 4 & 0,38 & 11931 & 11 & 0,44 & 13785 \\
Rhône-Alpes & 5 & 0,43 & 19300 & 3 & 0,53 & 23755 & 8 & 0,46 & 20568 \\
Champagne-Ardenne & 6 & 0,41 & 10610 & 11 & 0,25 & 6457 & 18 & 0,34 & 8815 \\
Franche-Comté & 7 & 0,35 & 5627 & 13 & 0,21 & 3464 & 10 & 0,45 & 7143 \\
Basse-Normandie & 7 & 0,35 & 6273 & 16 & 0,09 & 1620 & 21 & 0,26 & 4500 \\
Lorraine & 9 & 0,34 & 8091 & 8 & 0,33 & 7676 & 8 & 0,46 & 10788 \\
Poitou-Charentes & 10 & 0,28 & 7344 & 16 & 0,09 & 2411 & 17 & 0,37 & 9422 \\
Alsace & 11 & 0,27 & 2244 & 14 & 0,19 & 1573 & 7 & 0,47 & 3876 \\
Picardie & 12 & 0,25 & 4794 & 15 & 0,13 & 2530 & 6 & 0,55 & 10483 \\
Auvergne & 13 & 0,22 & 5763 & 12 & 0,24 & 6227 & 4 & 0,56 & 14625 \\
Bretagne & 14 & 0,19 & 5100 & 10 & 0,26 & 7157 & 14 & 0,40 & 10815 \\
Languedoc-Roussillon & 15 & 0,18 & 4927 & 1 & 0,89 & 24570 & 12 & 0,43 & 11840 \\
Ile-de-France & 16 & 0,09 & 1104 & 19 & 0,08 & 949 & 5 & 0,55 & 6559 \\
Nord-Pas-de-Calais & 17 & 0,08 & 969 & 16 & 0,09 & 1156 & 13 & 0,42 & 5147 \\
Aquitaine & 18 & 0,07 & 3119 & 5 & 0,37 & 15595 & 14 & 0,40 & 16374 \\
Midi-Pyrénées & 19 & 0,06 & 2549 & 20 & 0,03 & 1287 & 18 & 0,34 & 15618 \\
Provence-Alpes-Côte-d'Azur & 20 & 0,01 & 444 & 5 & 0,37 & 11569 & 16 & 0,39 & 12236 \\
Haute-Normandie & 20 & 0,01 & 147 & 21 & 0,02 & 221 & 20 & 0,29 & 3557 \\
Corse & 22 & 0 & 0 & 22 & 0 & 0 & 22 & 0,13 & 1111 \\
\hline
\end{tabular}



la prise en compte de données intermédiaires. En effet, le podium de 1793 est constitué des mêmes régions que celui de 2005, à savoir les régions Limousin, Pays-de-la-Loire et Centre-Val de Loire, même si l'ordre s'avère différent, alors que seule la région CentreVal de Loire figure sur celui de 1834. La capacité de résilience lentique de certaines régions va pouvoir ainsi être mise en avant par des observations court-termistes alors que la comparaison des inventaires extrêmes aurait pu nous laisser croire à une forme de linéarité.

\section{Les données numériques des inventaires}

Contrairement aux superficies, nous ne disposons pas de trois recensements pour étayer cette évolution géohistorique mais de deux, celui de 1834 ne produisant pas de chiffres. Si les fiabilités des recensements sur le plan surfacique permettaient de s'affranchir des distorsions liées à l'approximation de certaines données, celles sur le plan numérique sont plus problématiques. En effet, utiliser le terme de « fiable » pour qualifier les travaux de J.B. Rougier de la Bergerie (1819) est assez risqué tant il est possible de faire des remarques. L'utilisation des cartes de Cassini masquant les plus petits étangs et l'arrondi à 50 plans d'eau près sont en effet des écueils scientifiques importants. La comparaison temporelle (tableau 4) va donc pécher par sous-estimation chronique des objets lentiques en 1793.

Tableau 4 : Comparaison du nombre de plans d'eau par département entre 1793 et 2005

(D'après J.B. Rougier de la Bergerie et P. Bartout, inédit).

(B) (C) (D) : voir tableau 2.

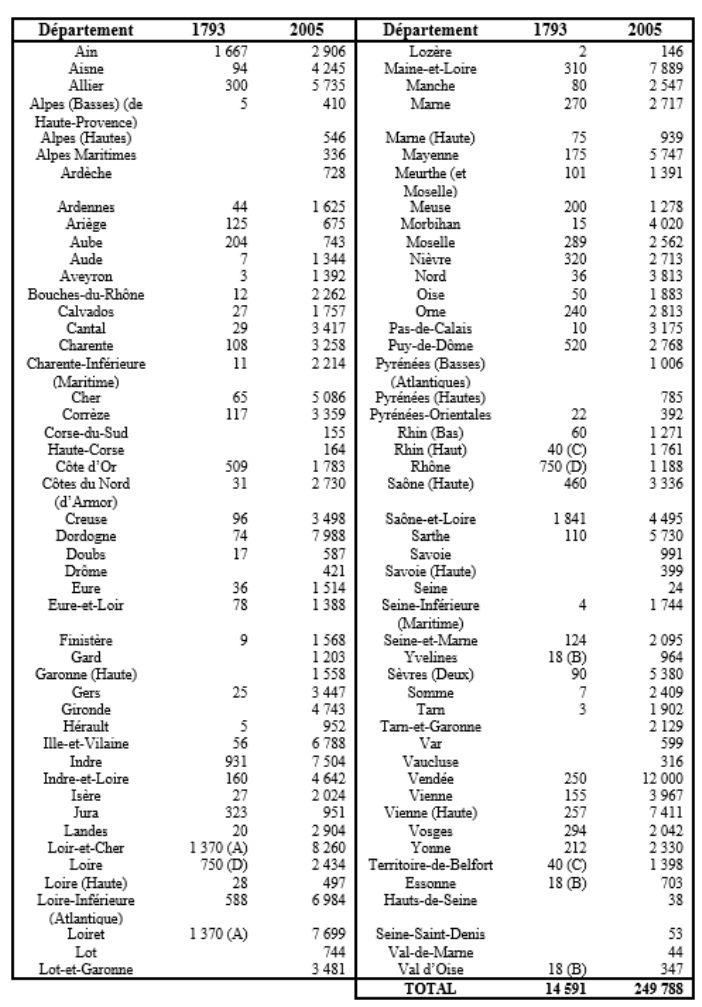

41

Si les augmentations de superficie entre 1793 et 2005 étaient globalement d'un rapport de un à trois, celles concernant le nombre de plans d'eau sont de un à dix-sept. La 
différence est extrêmement importante et sous entendrait la miniaturisation des plans d'eau durant le laps de temps écoulé entre les deux inventaires, même en tenant compte des lacunes de l'inventaire de 1793.

Toutes ces observations nécessitent donc un approfondissement méthodologique en cartographiant les nuances spatio-temporelles des localisations de plans d'eau au cours des deux derniers siècles en France.

\section{Cartographier les nuances spatio-temporelles de concentrations de plans d'eau en France}

43 Spatialiser des phénomènes et vérifier les correspondances avec les dynamiques nationales ou régionales permet de mieux comprendre son territoire limnique. De fait, cette dernière partie va s'attacher à produire des cartes de synthèse temporelles et thématiques à partir des critères développés jusqu'à présent pour identifier les dynamiques lentiques passées et actuelles.

\section{A. L'évolution des limnicités étendues en France depuis 1793}

44 A partir de l'état des lieux lentique de 1793 (figure 4), où la localisation préférentielle des fortes superficies d'étangs apparaît sous la forme d'une écharpe accrochée à Paris, balayant la totalité du sud et de l'est du Bassin Parisien, ainsi que des bordures métamorphiques des massifs armoricain et central, il nous est possible de cartographier l'évolution de la limnicité étendue au cours des deux derniers siècles.

Figure 4 : La stagnucité départementale en 1793.

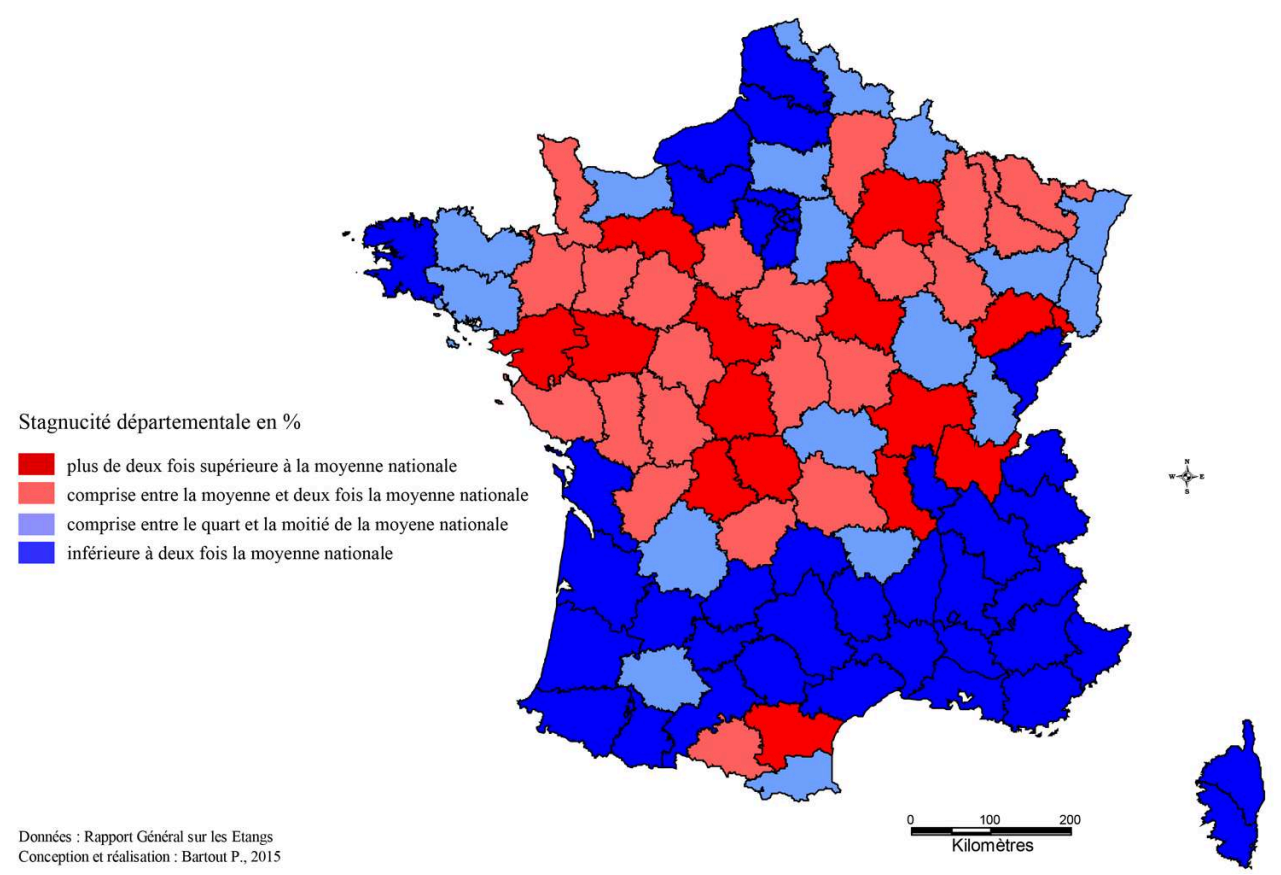

Comme l'analyse du tableau 3 a pu nous le démontrer, cette démarche va privilégier les tendances courtes (en comparant les inventaires se succédant historiquement) et non celles longues (en prenant les inventaires extrêmes). 


\section{L'évolution des stagnucités entre 1793 et 1834} gradient géographique, latitudinal avec deux secteurs (Nord et Sud) aux faibles stagnucités qui encadrent le secteur dominant (Centre), et longitudinal pour la partie centrale avec des très fortes stagnucités dans la partie ouest de la France et de plus faibles à l'Est, les tendances entrevues par l'intermédiaire du tableau 2 laissent penser à une remise en cause de cet état des lieux.

Figure 5 : L'évolution de la stagnucité départementale entre 1793 et 1834.

Evolution de la stagnucité départementale

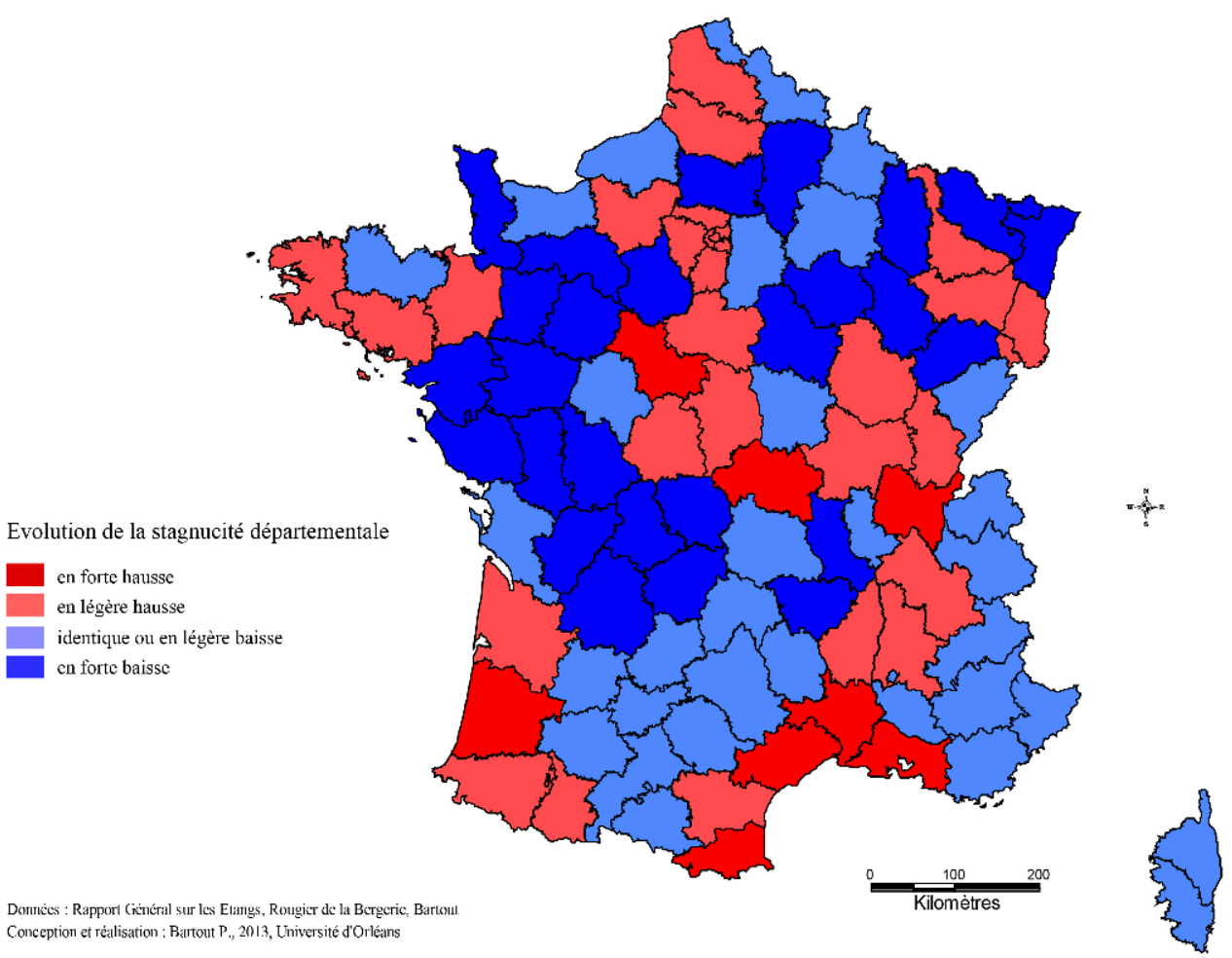

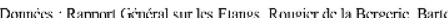
Conception et réallisation : Bartout P., 2013, Université d'Orléans

En dehors de la forte hausse déjà évoquée pour les côtes languedociennes et landaises, d'autres espaces connaissent une hausse de leur stagnucité post-Révolution: il s'agit notamment du Berry, de l'Orléanais, de la Bretagne ou de la plaine de la Saône. Mais l'élément le plus marquant de cette carte est la décroissance extrêmement importante touchant le Grand Ouest de la France, Bretagne exceptée (- $42 \%$ en Limousin, - 51 \% en Pays-de-la-Loire, - $67 \%$ en Poitou-Charentes et surtout - $74 \%$ en Basse-Normandie). Ces territoires ont pour un certain nombre souffert des guerres vendéennes et de la chasse aux signes appartenant aux ordres privilégiés (dont l'étang), mais la cause de ce phénomène est avant tout à rechercher du côté des structures agraires. Pays de bocages, de métayers, la disparition des superficies d'étangs est avant tout à remettre en parallèle avec le potentiel financier des nouveaux propriétaires issus de la vente des Biens Nationaux. Peu nombreux à pouvoir entretenir les grands étangs présents en 1793, les gestionnaires ont eu le choix entre la suppression complète de l'étang ou la réduction à une superficie plus en rapport avec leurs moyens financiers. 


\section{L'évolution des limnicités étendues entre 1834 et 2005}

Si les deux figures précédentes présentaient des résultats portant sur la stagnucité car, de fait, seuls les étangs étaient inventoriés, l'inventaire de 2005 s'appuie sur l'ensemble du corpus limnique et la comparaison temporelle va donc porter sur la limnicité étendue et non la seule stagnucité.

L'évolution entre 1834 et 2005 de cette limnicité étendue départementale montre une très forte différence avec la précédente, même si les résultats ne font pas apparaître les multiples variations internes à cette longue période temporelle allant du milieu du 19 ème siècle au tout début du $21^{\text {ème }}$ siècle.

Figure 6 : L'évolution de la limnicité étendue départementale entre 1834 et 2005.

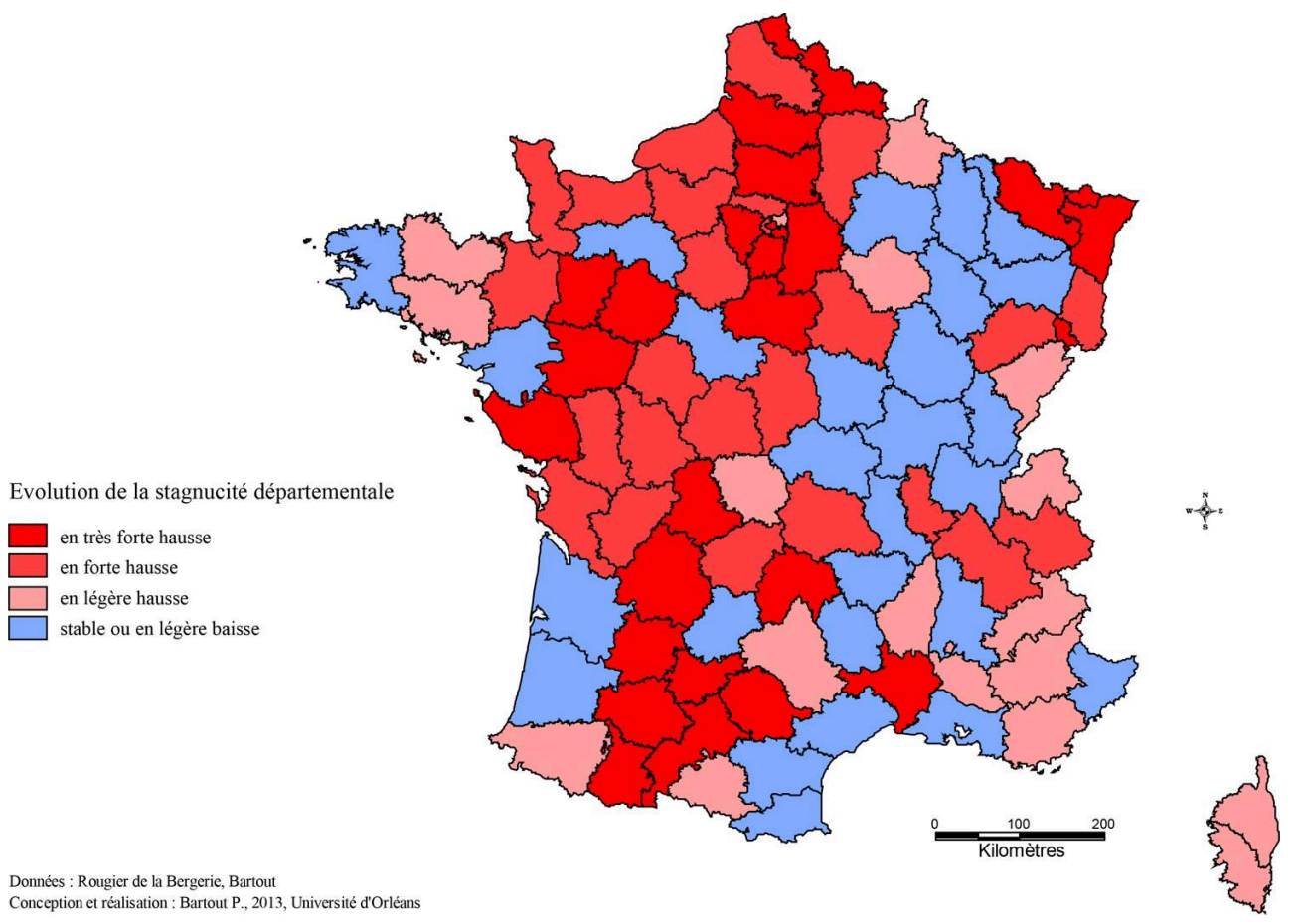

En premier lieu, la légende montre une différence notable avec les figures précédentes par l'absence de la classe des très fortes baisses : cela signifie que l'on assiste à une généralisation du plan d'eau touchant désormais toutes les contrées même les plus perméables et les plus urbanisées.

51 Le Grand Ouest (excepté encore une fois la Bretagne), mais aussi le Nord-ouest, la Région Parisienne et surtout le Midi toulousain connaissent une poussée des superficies de plans d'eau sans commune mesure dans l'histoire. En pourcentage, ce sont logiquement des régions peu couvertes en plans d'eau qui connaissent la plus forte progression (Corse, Haute-Normandie, Midi-Pyrénées ou Ile-de-France avec + $591 \%$ ). En revanche, le Grand Est (Champagne-Ardenne, Lorraine, Bourgogne) connaît une évolution différente par relative stagnation, expliquant son passage de région lentique principale à celle de secondaire aujourd'hui. Enfin, le Sud littoral et le Sud-est de la France en général sont en stagnation, voire en déclin. 


\section{Synthétiser la dynamique temporelle des limnicités étendues régionales}

Caractériser la dynamique temporelle des limnicités étendues, c'est comprendre pour un même territoire les variations en lien avec les dynamiques sociétales et les équilibres économiques et politiques recherchés.

Cette figure 7 fait clairement ressortir l'idée d'un « retard » lentique des Midis français, tout comme elle met en lumière la place historiquement importante de six régions (Centre-Val-de-Loire, Pays-de-la-Loire, Limousin, Bourgogne, Lorraine et Rhône-Alpes) qui ont, depuis 1793, toujours eu une stagnucité supérieure à la moyenne nationale. Enfin, les régions intermédiaires sont composées d'un axe central (Picardie, Ile-de-France, Auvergne) en plein développement des superficies de plans d'eau, et de deux parties périphériques qui peinent à retrouver leur lustre d'antan (BasseNormandie et Poitou-Charentes à l'Ouest; Champagne-Ardenne, Franche-Comté et Alsace à l'Est).

Figure 7 : L'évolution de la limnicité étendue régionale entre 1793 et 2005 en France.

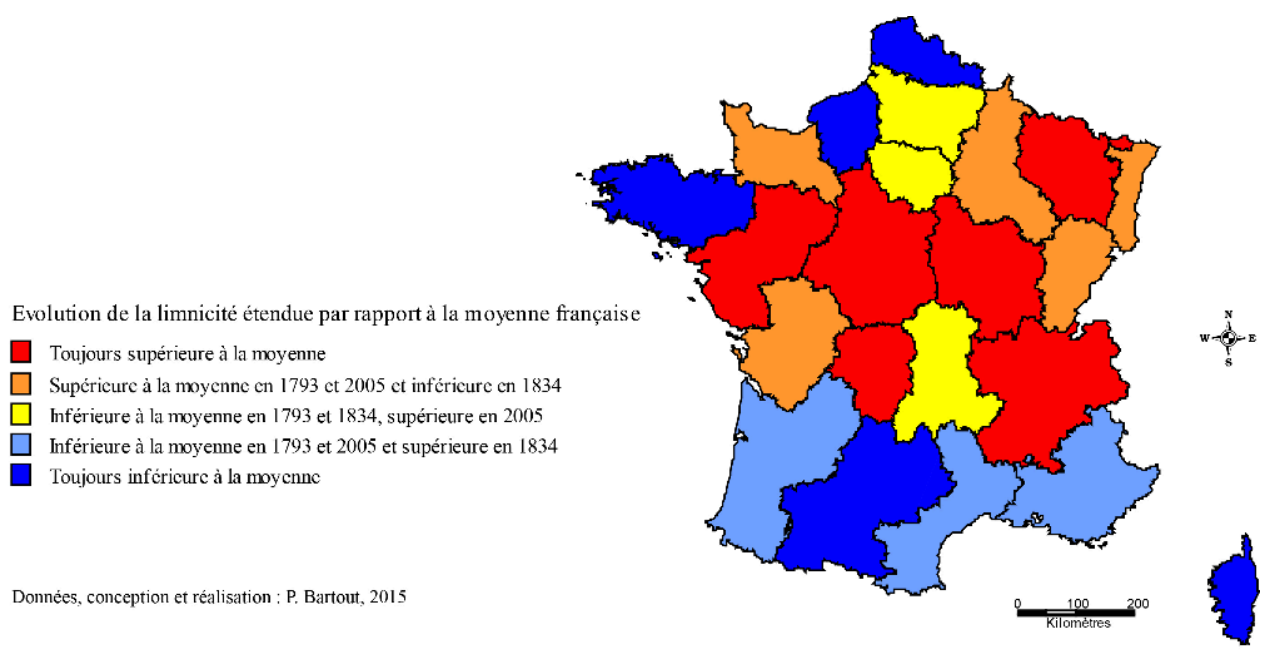

Mais se limiter à la seule approche surfacique est trop réducteur. Que disent alors les évolutions numériques même si nous ne pouvons attendre autant d'informations que pour la limnicité étendue du fait de l'absence de recensement intermédiaire?

\section{B. L'évolution des densités de plans d'eau en France depuis 1793}

La faiblesse de la validité des chiffres de 1793 que nous avons évoquée précédemment nous contraint de ne pas nous hasarder dans des représentations graphiques douteuses. Aussi, nous nous sommes contentés d'une carte de synthèse (figure 8) à l'échelle régionale de la permanence lentique entre 1793 et 2005. 
Figure 8 : L'évolution des densités de plans d'eau entre 1793 et 2005.

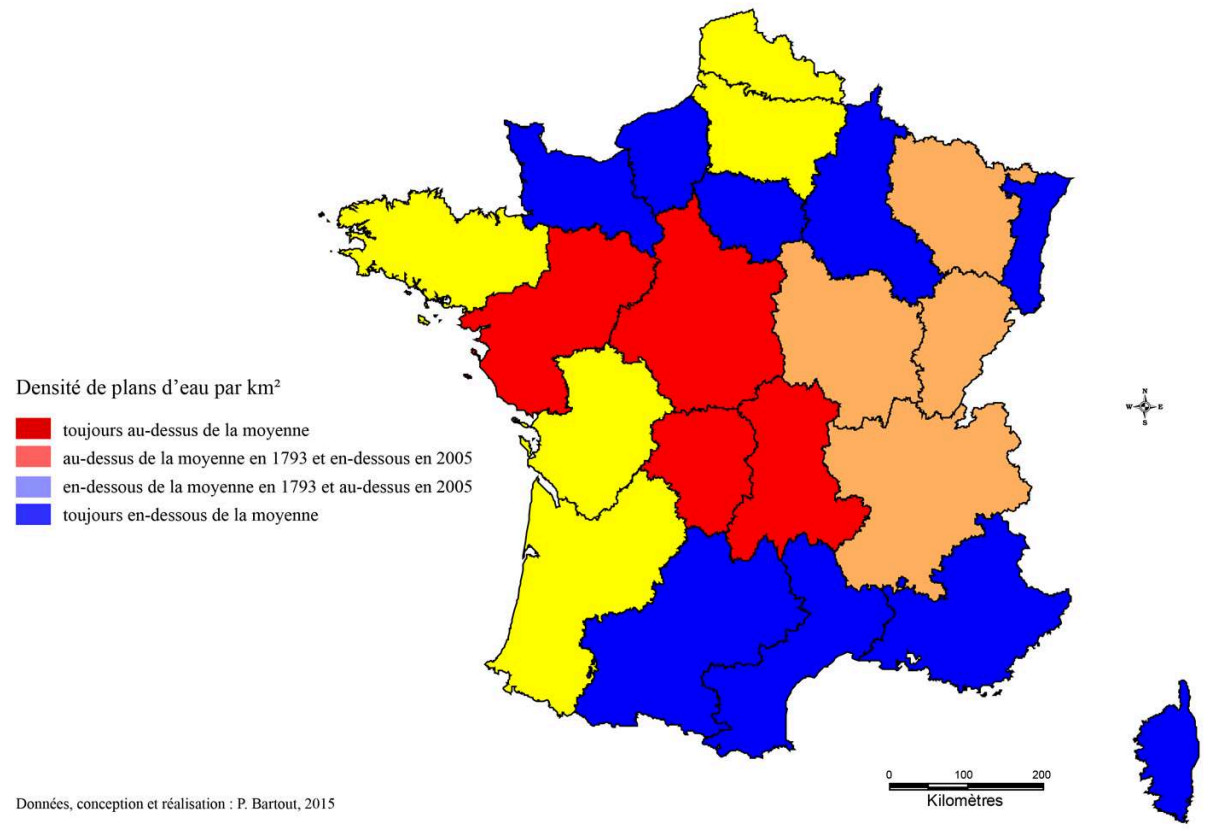
Auvergne) constitue l'élément central de l'édifice lentique puisque, en nombre, il a, depuis 1793, toujours hébergé des densités au-dessus des moyennes nationales. A ce pôle géographique premier était adjoint auparavant le Centre-est de la France qui se trouve être en net recul depuis quelques décennies (Rhône-Alpes, Bourgogne, FrancheComté, Lorraine). En revanche, un arc atlantique vient le supplanter depuis quelques temps (Aquitaine, Poitou-Charentes, Bretagne) sous l'effet de la mode des loisirs, auquel il s'agit d'adjoindre le Nord de la France (Picardie, Nord-Pas-de-Calais).

- une bande latitudinale allant de la Basse-Normandie jusqu'à l'Alsace (à l'exception de la Lorraine) où le nombre d'étangs augmente sans pour autant permettre à ces régions d'apparaître comme dominantes en France ;

- le pourtour méditerranéen accompagné de la région Midi-Pyrénées où le nombre d'étangs est très peu élevé et tend à se maintenir à des bas niveaux.

\section{Faire apparaître des dynamiques lentiques géographiques} plans d'eau artificiels en insistant sur les continuités et discontinuités de cette occupation par des populations distinctes, l'hétérogénéité des données n'a pas permis de quantifier efficacement leurs plans d'eau. Grâce aux données collectées à l'échelle du département, nous avons été en revanche en mesure de quantifier l'évolution statistique et spatiale des concentrations et superficies de plans d'eau au cours des deux derniers siècles. 
61 Par l'intermédiaire de la figure 9 ci-après, nous allons synthétiser les différentes informations collectées jusqu'à présent et montrer les dynamiques lentiques géographiques à l'échelle régionale.

Figure 9 : Les dynamiques lentiques depuis 1793 par région administrative en France.

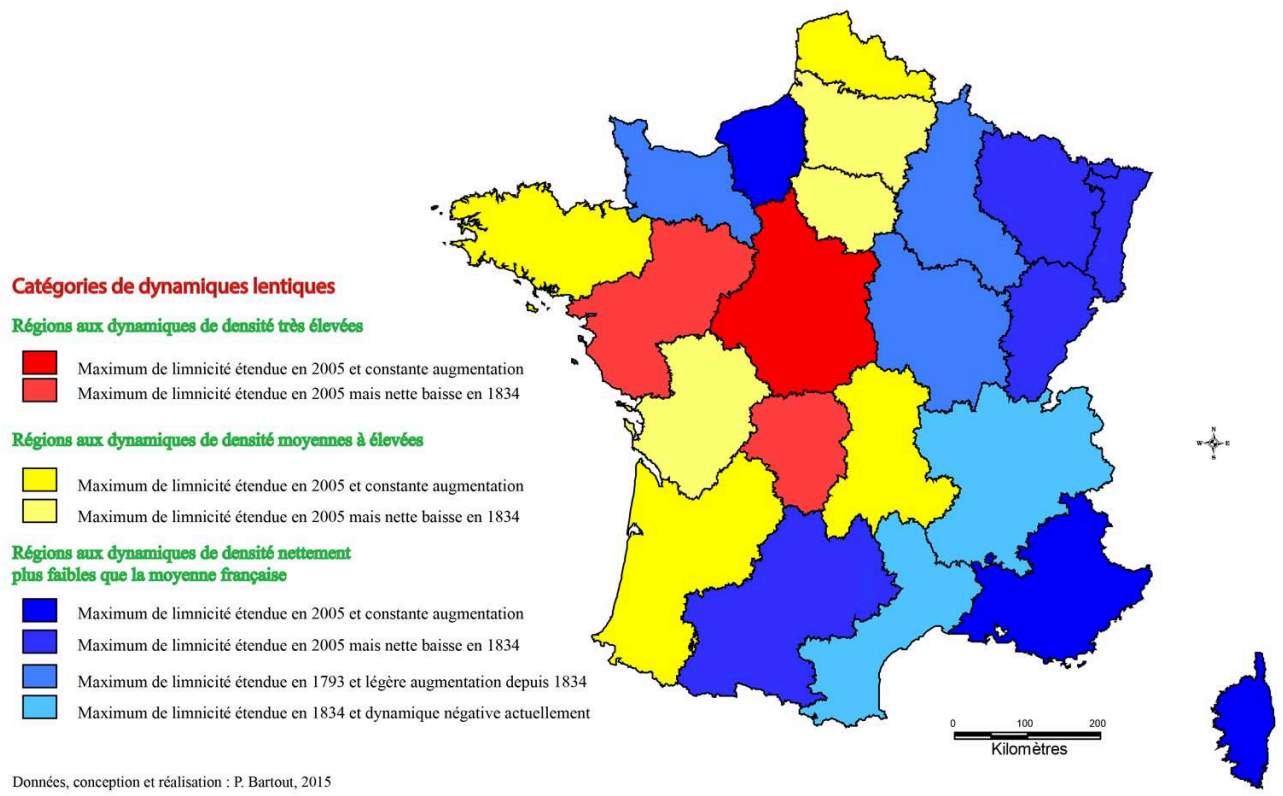

62 La France des plans d'eau étudiée à l'échelle régionale depuis 1793 procure graphiquement huit catégories de fonctionnements lentiques que nous pouvons regrouper en trois grands ensembles :

63 - les régions de premier plan (Centre-Val-de-Loire, Pays-de-la-Loire, Limousin) où la densité et la limnicité étendue ont toujours été supérieures à la moyenne lors des trois inventaires étudiés ;

64 - les régions (Auvergne, Aquitaine, Bretagne, Ile-de-France, Nord-Pas-de-Calais, Picardie, Poitou-Charentes) où les deux indicateurs (densité et limnicité étendue) sont en augmentation rapide à très rapide, faisant d'elles des régions émergentes, bien que certaines d'entre elles aient connus des accidents historiques ;

65 - les autres régions de plans d'eau, principalement situées à l'Est et au Sud, qui abritent aujourd'hui moins de plans d'eau que leurs consœurs occidentales tout en connaissant malgré tout une hausse de leur densité de plans d'eau. A l'intérieur de cet ensemble, nous avons distingué six fonctionnements différenciés :

- les régions traditionnelles de plans d'eau artificiels situées au Nord-est de la France (Lorraine, Bourgogne, Alsace, Champagne-Ardenne, Franche-Comté) qui continuent à abriter de grandes superficies d'étangs mais qui sont moins concernées par la multiplication des petits plans d'eau contemporains ;

- les deux régions normandes où la place importante des mares limite leur limnicité étendue, les distinguant de l'ensemble occidental français ;

- les régions (Poitou-Charentes, Champagne-Ardenne, Franche-Comté, Alsace) au passé lentique supérieur à la moyenne française qui ont régressé dans la hiérarchie nationale tout en voyant le fait « étang » progresser ces dernières décennies ; 
- la région Midi-Pyrénées qui voit ses paysages se couvrir localement d'étangs ces dernières années sans que les données n'atteignent les valeurs moyennes françaises ;

- les régions (Provence-Alpes-Côte-d'Azur et Corse) où malgré des dynamiques lentiques légèrement positives, le retard lentique est très important même si localement (sur les littoraux), de très fortes valeurs de limnicité étendue peuvent exister ;

- les régions Rhône-Alpes et Languedoc-Roussillon qui sont les seules à connaître une dynamique négative des limnicités étendues par suppression d'un certain nombre de grands plans d'eau au cours des $19^{\text {ème }}$ et $20^{\text {ème }}$ siècles.

\section{Conclusion}

Grâce aux multiples analyses statistiques présentées, nous avons pu montrer que les paysages de plans d'eau étaient sans cesse réécrits et adoptaient des trajectoires paysagères lentiques variées, faites de continuités et discontinuités historiques depuis l'épisode révolutionnaire en France.

Grâce à l'approche temporelle, nous avons pu comprendre s'il existait et existe encore aujourd'hui des régions où la prégnance numérique, spatiale et psychologique des étangs a pu marquer le territoire et ses habitants, au point de lui conférer une image stagnustre construite, qui serait à même de produire un périmètre de gestion idoine actuellement.

En effet, l'approche actuelle faite par l'intermédiaire des « hydro-écorégions » (Wasson $\& a l, 2004)$ permet certes la distinction de milieux au potentiel naturel très inégal quant aux possibilités d'édification d'une retenue d'eau, mais le géosystème centré sur le plan d'eau artificiel et notamment l'étang ne peut être appréhendé par sa seule variable continue : ce socle naturel doit être complété par une variable d'entrée discontinue du système et celle-ci repose sur les trajectoires paysagères issues de paramètres sociaux, économiques et historiques car l'étang résulte des arbitrages sociétaux d'une époque donnée, sa présence pouvant donc être éphémère, voire pas du tout souhaitée.

Les différentes analyses graphiques et statistiques produites ont permis de faire apparaître la domination stagnustre actuelle et passée de trois régions (Centre-Val-deLoire, Pays-de-la-Loire et Limousin). Cette continuité, cette capacité à se maintenir à de hauts niveaux stagnustres (Centre-Val-de-Loire) ou à rebondir (Pays-de-la-Loire, Limousin) après des accidents historiques en proposant des sites géographiques variés adaptés aux demandes sociétales évolutives, doivent constituer des critères appréciables pour donner à ces trois régions une légitimité nationale en termes de fonctionnement, de gestion, de répartition et d'impacts sur et par le milieu.

\section{BIBLIOGRAPHY}

Abbé J-L., 2007, « Qui assèche un étang au Moyen-âge ? Société et bonification des milieux humides en Languedoc », Aestuaria, p. 49-64. 
Balabanian O., Bouet G., 1989, L'eau et la maîtrise de l'eau en Limousin, Treignac, Les Monédières, 296 p.

Bartout P., 2006, Pour un référentiel des zones humides intérieures de milieu tempéré : l'exemple des étangs en Limousin (France) - Typologies, Régionalisation., Thèse de doctorat en géographie, Université de Limoges, $497 \mathrm{p}$.

Bartout P., 2010, Pour un référentiel des zones humides intérieures de milieu tempéré - l'exemple des étangs en Limousin (France), Sarrebruck, Editions Universitaires Européennes, 456 p.

Bartout P., 2011, « L'apport du cadastre napoléonien aux problématiques spatiales des retenues d'eau », Revue Géographique de l'Est, 16 p.

Bartout P., 2012, Les étangs du Limousin : des zones humides face au développement durable, Brive-laGaillarde, Les Monédières, 250 p.

Bartout P., 2015a, « L'incompréhension de la place prise par les plans d'eau dans l'Union Européenne et ses conséquences règlementaires », Norois, 235, p. 17-36.

Bartout P., 2015b, Les territoires limniques. Nouveau concept limnologique pour une gestion géographique des milieux lentiques, Mémoire HDR de géographie, Université d'Orléans, 444 p.

Bartout P., 2015c, « Un potentiel lentique méconnu : la France aux 250000 étangs », in Touchart L., Bartout P., Motchalova O. (dir), Mieux comprendre les étangs : expériences nationales et internationales, du Berry Limousin à l'Europe orientale, Brive-la-Gaillarde, Les Monédières, p. 27-49.

Bartout P., Bernard C., 2007, « Peut-on concevoir la définition de l'étang comme un outil de gestion? ", in Touchart L. (dir), Géographie de l'étang : des théories globales aux pratiques locales, Paris, L'Harmattan, p. 87-100.

Bartout P., Touchart L., 2013, «L'inventaire des plans d'eau français : outil d'une meilleure gestion des eaux de surface », Annales de Géographie, 691, p. 266-289.

Bédoucha G., 2011, Les liens de l'eau : en Brenne, une société autour des étangs, Paris - Versailles, Maison des Sciences de l'homme et Quæ, 688 p.

Bénarrous R., 2003, « La Brenne des étangs : une zone humide créée au Moyen-âge »,Zones Humides Infos, 42, p. 2-3.

Bénarrous R., 2009, La grande Brenne aux périodes préindustrielles : contribution à l'histoire des paysages, des étangs et des relations sociétés/milieux dans une zone continentale : approches historique, archéologique et paléo environnementale, Thèse de doctorat, Université Panthéon Sorbonne de Paris, $605 \mathrm{p}$.

Bénarrous R., Marinval M-C., 2003, «Carpes et zones d'étangs, des histoires étroitement liées », Zones Humides Infos, 42, p. 3-4.

Benoît C., 1992, Les étangs de la Dombes au Moyen-âge, XIIIème-XVème siècles, Besançon, Comité des Travaux historiques et scientifiques, $102 \mathrm{p}$.

Bernard C., 2008, L'étang, l'homme et l'oiseau : Incidences des modes de gestion des étangs piscicoles sur les ceintures de végétation et l'avifaune nicheuse en Sologne, Brenne, Bresse, Territoire de Belfort et Champagne humide, Thèse de doctorat en géographie, ENS Lyon, $630 \mathrm{p}$.

Berque A., 1996, Etre humains sur la Terre, principes d'éthique de l'écoumène, Paris, Gallimard, 212 p. Blouin-Gourbilière C., 2013, L'élaboration d'images « paysages » habitantes: un levier participatif d'aménagement du territoire. Le cas du Parc Naturel Régional de la Brenne, Thèse de doctorat en Géographie, Université d'Angers - Agrocampus Ouest, 568 p. 
Bouleau G., Pont D., 2014, « Les conditions de référence de la directive cadre européenne sur l'eau face à la dynamique des hydrosystèmes et des usages ", Natures Sciences Sociétés, 22, p. 3-14.

Bravard J.-P., Pourtier R., 2003, « Les territoires de l'eau. Introduction », Bulletin de l'Association de Géographes Français, 80(3), p. 239-241.

Capdepuy V., Djament-Tran G., 2012, « Qu'est-ce que la géohistoire ? », Carnet de géohistoire.

Cassan M., 2006, « Etangs et Révolution : l'application du décret d'assèchement des étangs du 14 frimaire an II (4 décembre 1793) dans le Limousin et la Marche », GHZH, p. 85-102.

Clément V., 1999, « Pays et paysages forestiers de Vieille Castille (XIe-XXe siècle) », Annales de Géographie, 108, p. 651-655.

Corboz A., 2001, Le Territoire comme palimpseste et autres essais, Besançon, Editions de l'Imprimeur, $284 \mathrm{p}$.

Derex J-M., 2001, La gestion de l'eau et des zones humides en Brie (fin de l'Ancien Régime - fin du XIXè siècle), Paris, L'Harmattan, 553 p.

Dufour S., Piégay H., 2009, "From the myth of a lost paradise to targeted river restoration: forget natural references and focus on human benefits", River Research and Applications, 25, p. 568-581.

Durand M-D., 1993, Le passé des étangs creusois, Mémoire de Maîtrise de géographie, Université de Limoges, $134 \mathrm{p}$.

Finger-Stich A., 2013, « Etangs en société : sujets versatiles », in Oertli B., Frossard P.-A. (dir), Mares et étangs : écologie, gestion, aménagement et valorisation, Lausanne, Presses Polytechniques et universitaires romandes, p. 13-20.

Forel F.-A., 1892, Le Léman, monographie limnologique, Lausanne, F. Rouge, 3 tomes, rééd. 1969 et 1998, Genève, Slatkine.

Franchomme M., 2008, Du cadastre napoléonien à la trame verte, le devenir des petites zones humides périurbaines en région Nord-Pas de Calais, Thèse de Doctorat en géographie, Université de Lille, 406 p.

Genest C., 2007, «La définition de l'étang en géographie linguistique », in Touchart L. (dir), Géographie de l'étang: des théories globales aux pratiques locales, Paris, L'Harmattan, p. 55-85.

Germain A., 2005, Etude géohistorique des étangs dans la Montagne limousine, Mémoire de Master 2, Université Paris I Panthéon, 86 p.

Ghiotti S., 2007, Les territoires de l'eau, gestion et développement en France, Paris, éd. CNRS, 247 p.

Grataloup C., 1996, Lieux d'histoire : essai de géohistoire systématique, Montpellier, Reclus, 200 p.

Hatton-Ellis T., 2010, “The hitchhiker's guide to the water framework directive”, Aquatic Conservation: Marine and Freshwater Ecosystems, 18, p. 111-116.

Jacob-Rousseau N., 2009, « Géohistoire / Géo-histoire : quelles méthodes pour quel récit ? », Revue de géographie de Lyon, 84(4), p. 209-288.

Kirat T., Torre A., 2008, Territoires de conflits : analyses des mutations de l'occupation de l'espace, Paris, L'Harmattan, $322 \mathrm{p}$.

Kolar S., 1991, Les étangs dans le territoire de Belfort, Mémoire de Maîtrise de géographie, Université de Besançon, $191 \mathrm{p}$.

Laganier R., Salvador P.-G., 2001, « Editorial », in Actes du colloque Hydrosystèmes, paysages, territoires, Lille, Université des Sciences et Technologies de Lille, p. 1-2. 
Landou N., 2006, « Les Célestins des Ternes et leurs étangs à l'époque moderne », GHZH, p. 51-66. Lespez L., 2012, Paysages et gestion de l'eau. Sept millénaires d'histoire de vallées et de plaines littorales en Basse-Normandie, Caen, PU Caen - MRSH, 336 p.

Lévy J., Lussault M., 2003, Dictionnaire de la géographie et de l'espace des sociétés, Paris, Belin, 1033 p. Lutz M., 2001, Les étangs de pisciculture en Europe Centrale. Typologie des systèmes d'exploitation et impacts des modalités de gestion sur l'avifaune, Thèse de Doctorat en géographie, Université de Strasbourg I, $213 \mathrm{p}$.

Marache C., 2007, « Un nouveau visage pour la Double : l'assainissement et le désenclavement d'une région humide méconnue au XIXè siècle », Aestuaria, p. 207-223.

McCormick J., 2001, Environmental Policy in the European Union, Basingstoke, Palgrave, 329 p.

Miras Y., 2004, L'analyse pollinique du plateau de Millevaches (Massif Central) et de sites périphériques limousins et auvergnats : approches des paléoenvironnements, des systèmes agro-pastoraux et évolution des territoires ruraux, Thèse de doctorat de Sciences et Vie, Université de Franche-Comté, 301 p.

Moine A., 2005, « Le territoire comme un système complexe pour l'aménagement et la géographie », Septièmes rencontres de Théo Quant.

Moss B., 2008, "The water framework directive: total environment or political compromise?", The Science of the Total Environment, 400, p. 32-41.

Pelletier M., 2002, Les cartes de Cassini : la science au service de l'Etat et des régions, Paris, Editions du Comité des Travaux Historiques et Scientifiques, $338 \mathrm{p}$.

Pitte J.-R., 1989, Histoire du paysage français, Paris, Tallandier, 447 p.

Rapport général sur les étangs, fait au Comité d'Agriculture et des Arts, par la Commission d'Agriculture et des Arts, an III, imp. « La feuille du cultivateur », 133 p.

Reyt P., 1998, Formes et paysages de l'eau dans le bassin de la Loire. De l'eau dans les paysages aux paysages de l'eau, Villeneuve d'Ascq, Presses Universitaires du Septentrion, 314 p.

Rougier de la Bergerie J-B (baron), 1819, Manuel des étangs ou traité de l'art d'en construire avec économie et solidité, Paris, Audot libraire, 198 p.

Sajaloli B., 1993, Les zones humides Laonnoises : fonctionnement, usages et gestion, Thèse de Doctorat de géographie, Université Paris I, $734 \mathrm{p}$.

Surridge B., Watson N., 2012, “Water Framework Directive”, in Bengtsson L., Herschy R.W., Fairbridge R.W. (dir), Encyclopedia of lakes and reservoirs, Dordrecht, Springer, p. 872-876.

Touchart L., 2014, Les territoires de l'eau en Russie, Paris, L'Harmattan, 331 p.

Vacher A., 1908, Le Berry, contribution à l'étude géographique d'une région française, Paris, Armand Colin, $548 \mathrm{p}$.

Vanier M., 2008, Le pouvoir des territoires. Essai sur l'interterritorialité, Paris, Anthropos, Economica, $158 \mathrm{p}$.

Wallerstein I.M., 1991, Geopolitics and geoculture: essays on the changing world-system, Paris, Maison des Sciences de l'Homme, $242 \mathrm{p}$.

Wasson J.G., Chandesris A., Pella H., Blanc L., 2004, « Les hydro-écorégions : une approche fonctionnelle de la typologie des rivières pour la Directive cadre européenne sur l'eau », Ingénieries-Eau Agriculture Territoires, 40, p. 3-12. 


\section{NOTES}

1. Un territoire limnique est « un territoire où les interrelations hommes - milieux - plans d'eau permettent d'identifier un espace aux caractéristiques limniques spatio-temporelles proches, offrant la possibilité, dans une optique de gestion, d'apporter une unité géographique, ceci à des échelles fluctuantes selon le facteur dominant envisagé » (Bartout, 2015b).

2. Pour A. Berque (1996), l'œkoumène (ou écoumène) désigne la relation de l'humain à son milieu : à la fois sensible et concrète, symbolique et technique.

3. C'est le cas notamment des travaux d'O. Balabanian et G. Bouet en Limousin en 1989, de nos travaux sur ce même Limousin en 2006 et 2012, et plus encore de C. Bernard en 2008 avec une approche innovante sur cinq régions d'étangs françaises (Brenne, Sologne, Bresse, ChampagneHumide et Territoire-de-Belfort) centrée sur la thématique de l'Oiseau.

4. Du moins ceux correspondant à la date de la campagne photogrammétrique.

5. C'est-à-dire la part en eau d'étang d'un territoire.

6. Part en eau dormante d'un territoire.

\section{ABSTRACTS}

Presently water frameworks, whether it is those European or French one, undervalue the part of societies in artificial lentic landscapes modelling. By a geohistorical approach which is pioneer in limnology, palimpsest characteristic of French limnic territories can be demonstrated. It is the fruit of many interactions between humans, environment and waterbodies. The use of three national inventories (1793, 1834 and 2005) allows bettering understanding the pond's concentrations (areas and numerous) since the end of the $18^{\text {th }}$ century, while engaging to explain the quantitative and qualitative variations noticed. The maps stemming from those analysis, for the most part unpublished, bring, at regional scale, valued information's out the temporal continuity of pond's localizations, as the surfacic and numeric superiority of the western central part of France (administrative regions of "Centre-Val-de-Loire", "Pays-de-la-Loire" and "Limousin") since two centuries in spite of their tumultuous histories, even though they put up only two important sites (the "Sologne" and the "Brenne"). The size of the pond's dispersal is in this way demonstrated over time and not only actually as some organisms of water's management affirm, indicating a human/water relationship sociologically and economically fixed in a territory.

Les politiques de l'eau actuelles, qu'elles soient européennes ou françaises, mésestiment grandement la part des sociétés dans le modelage des paysages lentiques artificiellement créés. Grâce à une approche géohistorique novatrice en limnologie, le caractère palimpseste des territoires limniques français va être démontré, fruit d'interactions multiples entre hommes, milieux et plans d'eau. L'utilisation de trois inventaires nationaux scientifiquement validés (1793, 1834 et 2005) permet de mieux comprendre les concentrations d'étangs (en superficie et en nombre) depuis la fin du $18^{\text {ème }}$ siècle, tout en s'attachant à expliquer les variations quantitatives et qualitatives observées. Les cartographies issues de ces analyses, pour la plupart inédites, font apparaître, à l'échelle régionale et départementale, des informations précieuses quant à la continuité temporelle des localisations d'étangs, comme la domination surfacique et numérique du Centre-Ouest de la France (régions Centre-Val-de-Loire, Pays-de-la-Loire et Limousin) depuis 
deux siècles malgré des histoires tumultueuses et variées, alors même qu'elles n'hébergent localement que deux zones identifiées d'importance nationale ou internationale (la Sologne et la Brenne). L'importance du mitage stagnustre est ainsi démontrée sur la durée et pas seulement actuellement comme le prétendent certains organismes de gestion de l'eau, dénotant un rapport Homme/Eau ancré sociologiquement et économiquement dans le territoire.

Die gegenwärtigen politischen Strömungen in der Wasserwirtschaft - sei es auf europäischer oder französischer Ebene - unterschätzen die Komponente menschlicher Gesellschaften auf die Formgestaltung künstlicher Stehgewässer in eklatanter Weise. Dank einer in der Limnologie neuartigen geohistorischen Betrachtungsweise, wird hier der palimpsestartige Charakter der französischen Gewässer als Ergebnis vielfacher Wechselwirkungen zwischen Mensch, Umwelt und Wasserflächen aufgezeigt werden. Die Verwendung dreier nationaler Inventare, die wissenschaftlichen Ansprüchen standhalten (1793, 1834 und 2005), ermöglicht ein besseres Verständnis der seit Ende des 18. Jahrhunderts (hinsichtlich Fläche und Anzahl) gestiegenen Dichte von Weiherflächen in Frankreich; Ziel ist dabei gleichzeitig eine Erklärung der beobachteten quantitativen wie qualitativen Schwankungen. Die aus diesen Analysen hervorgegangenen und meist unveröffentlichten Kartierungen lassen auf Ebene der Regionen wie Départements wertvolle Informationen zu Tage treten, welche die Lagestabilität von Weiherflächen im Lauf der Zeit betreffen; es zeigt sich so bspw. eine seit zwei Jahrhunderten hinsichtlich Oberfläche und Anzahl (der stehenden Wasserflächen) bestehende Dominanz des westlichen Zentralfrankreich (Regionen Centre-Val-de-Loire, Pays-de-la-Loire und Limousin), und dies trotz aller Stürme und Wirren der Geschichte und obwohl diese Regionen nur zwei Gebiete beinhalten, die als national und international bedeutend eingestuft werden (Sologne und Brenne). Die Bedeutung der Zerstückelung der Flächen der Stehgewässer wird somit auch in diachroner Sicht aufgezeigt und nicht nur in synchroner Hinsicht, wie es in der Darstellung gewisser Stellen in der Wasserwirtschaftsverwaltung zum Ausdruck kommt, die auf einen Zusammenhang zwischen Mensch und Wasser verweisen, der soziologisch und ökonomisch im Gebiet verankert ist.

\section{INDEX}

Schlüsselwörter: Teich, Wechselwirkung, Gebiet, Inventar, Kartierung, Belastbarkeit, Entwicklung

Keywords: ponds, interactions, territory, inventory, maps, evolution, resiliency

Mots-clés: étangs, interactions, territoire, inventaires, cartographie, évolution, résilience

\section{AUTHOR}

\section{PASCAL BARTOUT}

Maître de conférences-HDR en géographie,ITP HCS, EA 1210 CEDETE,Université d'Orléans. pascal.bartout[chez]univ-orleans.fr 